TRANSACTIONS OF THE

AMERICAN MATHEMATICAL SOCIETY

Volume 365, Number 1, January 2013, Pages 213-250

S 0002-9947(2012)05586-3

Article electronically published on August 30, 2012

\title{
DIMENSION OF SLICES THROUGH THE SIERPINSKI CARPET
}

\author{
ANTHONY MANNING AND KÁROLY SIMON
}

\begin{abstract}
For Lebesgue typical $(\theta, a)$, the intersection of the Sierpinski carpet $F$ with a line $y=x \tan \theta+a$ has (if non-empty) dimension $s-1$, where $s=\log 8 / \log 3=\operatorname{dim}_{\mathrm{H}} F$. Fix the slope $\tan \theta \in \mathbb{Q}$. Then we shall show on the one hand that this dimension is strictly less than $s-1$ for Lebesgue almost every $a$. On the other hand, for almost every $a$ according to the angle $\theta$-projection $\nu^{\theta}$ of the natural measure $\nu$ on $F$, this dimension is at least $s-1$. For any $\theta$ we find a connection between the box dimension of this intersection and the local dimension of $\nu^{\theta}$ at $a$.
\end{abstract}

\section{INTRODUCTION}

Let $F$ denote the Sierpinski carpet [14, p.81] in the unit square $[0,1] \times[0,1]=I^{2}$ in the plane $\mathbb{R}^{2}$ and let $E_{\theta, a}:=\{(x, y) \in F: y-x \tan \theta=a\}$ denote its intersection with the line segment Line $\theta, a$ across $I^{2}$ of slope $\theta$ through $(0, a)$. (We only consider $\theta \in\left[0, \frac{\pi}{2}\right)$ because the case $\theta \in\left[\frac{\pi}{2}, \pi\right)$ is equivalent under the transformation $(x, y) \mapsto(x, 1-y)$.) We shall study the dimension of $E_{\theta, a}$ for $a \in I^{\theta}:=[-\tan \theta, 1]$, as a subset of the $y$-axis.
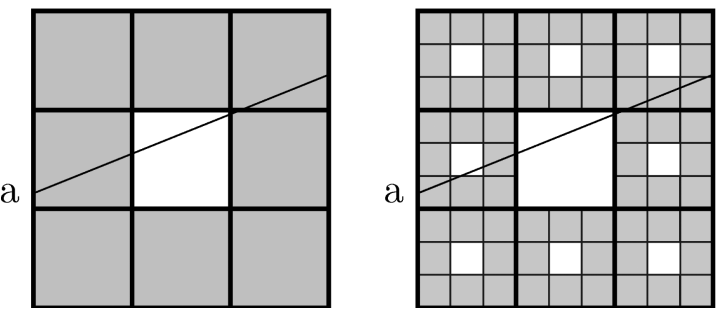

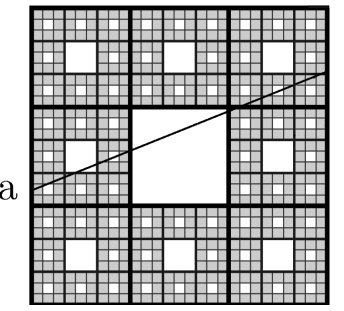

FiguRE 1. The intersection of the Sierpinski carpet with the line $y=\frac{2}{5} x+a$ for some $a \in[0,1]$.

The angle $\theta$ projection of the unit square $[0,1]^{2}$ to the $y$-axis is

$$
\operatorname{proj}^{\theta}(x, y):=(-\tan \theta, 1) \cdot(x, y) \text {. }
$$

When $\tan \theta \in \mathbb{Q}$, we shall show as our main result, in Theorem 9, that for Lebesgue almost every $a$,

$$
\operatorname{dim}_{\mathrm{H}} E_{\theta, a}<\log 8 / \log 3-1 .
$$

Received by the editors September 3, 2009 and, in revised form, January 24, 2011.

2010 Mathematics Subject Classification. Primary 28A80; Secondary 37H15, 37C45, 37B10, $37 \mathrm{~A} 30$

Key words and phrases. Self-similar sets, Hausdorff dimension, dimension of fibres.

This research was supported by the Royal Society grant 2006/R4-IJP and the research of the second author by the OTKA Foundation \#T 71693.

(C)2012 American Mathematical Society Reverts to public domain 28 years from publication 
This behavior for all rational slopes is atypical because, as an easy consequence of some results of Marstrand (see [12, Chapter 10]), we shall prove in Lemma 2 that, for Lebesgue almost all $(\theta, a), \operatorname{dim}\left(E_{\theta, a}\right)=s-1$, where $s=\log 8 / \log 3=\operatorname{dim}_{\mathrm{H}} F$.

When $\tan \theta \in \mathbb{Q}$ the opposite behavior also occurs because, for $\nu^{\theta}$-almost all $a \in I^{\theta}$, we shall show $\operatorname{dim}\left(E_{\theta, a}\right) \geq s-1$. Here $\nu^{\theta}$ is defined as follows.

We order the vectors $(u, v) \in\{0,1,2\} \times\{0,1,2\} \backslash\{(1,1)\}$ in lexicographic order and write $\mathbf{t}_{i}$ for the $i$-th vector, $i=1, \ldots, 8$. The Sierpinski carpet $F$ is the attractor of the IFS

$$
\mathcal{G}:=\left\{g_{i}(x, y)=\frac{1}{3}(x, y)+\frac{1}{3} \mathbf{t}_{i}\right\}_{i=1}^{8} .
$$

Let $\Sigma_{8}:=\{1, \ldots, 8\}^{\mathbb{N}}$ and write $\sigma: \Sigma_{8} \rightarrow \Sigma_{8}$ for the left shift. We write $\Pi: \Sigma_{8} \rightarrow F$ for the natural projection

$$
\Pi(\mathbf{i}):=\lim _{n \rightarrow \infty} g_{i_{1} \ldots i_{n}}(0)
$$

and $\nu:=\Pi_{*} \mu_{8}$ for the natural measure on $F$, where $\mu_{8}$ is the Bernoulli measure on $\Sigma_{8}$ given by $\left\{\frac{1}{8}, \ldots, \frac{1}{8}\right\}^{\mathbb{N}}$. Then $\nu^{\theta}:=\operatorname{proj}_{*}^{\theta}(\nu)$.

Feng and Hu proved [4, Theorem 2.12] that every self-similar measure $\eta$ is exact dimensional. That is, the local dimension of the measure $\eta$, given by

$$
d(\eta, x)=\lim _{r \rightarrow 0} \frac{\log \eta(B(x, r))}{\log r},
$$

is defined and constant for $\eta$-almost all $x$. It was shown by Young 19] that this constant must be the Hausdorff dimension of the measure $\eta$. That is,

$$
\text { for } \eta \text { a.a. } x, d(\eta, x)=\operatorname{dim}_{\mathrm{H}}(\eta):=\inf \left\{\operatorname{dim}_{\mathrm{H}}(U): \eta(U)=1\right\} .
$$

We will apply this result to the measure $\nu^{\theta}$, which is a self-similar measure for the IFS

$$
\Phi:=\left\{\varphi_{i}^{\theta}(t)=\frac{1}{3} \cdot t+\frac{1}{3} \cdot \operatorname{proj}^{\theta}\left(\mathbf{t}_{i}\right)\right\}_{i=1}^{8}
$$

with equal weights. That is, for every Borel set $B$,

$$
\nu^{\theta}(B)=\sum_{k=1}^{8} \frac{1}{8} \nu^{\theta}\left(\left(\varphi_{k}^{\theta}\right)^{-1}(B)\right) .
$$

Since $\nu^{\theta}$ is a self-similar measure on $I^{\theta} \subset \mathbb{R}$, (3) gives

$$
\text { for } \nu^{\theta} \text { a.a. } x, d\left(\nu^{\theta}, x\right) \leq 1 \text {. }
$$

\section{Statement of Results}

2.1. Behavior for typical slope. A special case of a theorem of Marstrand [13] (also see [12, Theorem 10.11]) is

Proposition 1 (Marstrand). For $\nu$-almost all $z \in F$ and for Lebesgue almost all $\theta \in[0, \pi)$ we have

$$
\operatorname{dim}\left(F \cap\left(z+W^{\theta}\right)\right)=s-1 \text { and } \mathcal{H}^{s-1}\left(F \cap\left(z+W^{\theta}\right)\right)<\infty,
$$

where $W^{\theta}$ is the straight line of angle $\theta$ through the origin. 
Lemma 2. For Lebesgue almost all $(\theta, a), a \in I^{\theta}, \theta \in[0, \pi / 2)$,

$$
\operatorname{dim}\left(E_{\theta, a}\right)=s-1,
$$

where dim denotes either $\operatorname{dim}_{\mathrm{H}}$ or $\operatorname{dim}_{\mathrm{B}}$.

Proof. This follows from [12, Theorem 10.10] using a density point argument.

2.2. The general case. We shall prove that for all $\theta$ the various dimensions are $\nu^{\theta}$-almost everywhere constant functions.

Proposition 3. Fix an arbitrary $\theta \in[0, \pi / 2)$. Then there exist non-negative numbers $d_{H}^{\theta}$ and $d_{\underline{B}}^{\theta}, d_{\bar{B}}^{\theta}$ such that for $\nu^{\theta}$-almost all $a \in I^{\theta}$ we have

$$
\operatorname{dim}_{\mathrm{H}}\left(E_{\theta, a}\right)=d_{H}^{\theta}, \underline{\operatorname{dim}}_{\mathrm{B}}\left(E_{\theta, a}\right)=d_{\underline{B}}^{\theta} \text { and } \overline{\operatorname{dim}}_{\mathrm{B}}\left(E_{\theta, a}\right)=d_{\bar{B}}^{\theta} .
$$

Proposition 4. For all $\theta \in[0, \pi / 2)$ and $a \in I^{\theta}$ if either of the two limits

$$
\operatorname{dim}_{\mathrm{B}}\left(E_{\theta, a}\right)=\lim _{n \rightarrow \infty} \frac{\log N_{\theta, a}(n)}{\log 3^{n}}, \quad d\left(\nu^{\theta}, a\right)=\lim _{\delta \rightarrow 0} \frac{\log \left(\nu^{\theta}[a-\delta, a+\delta]\right)}{\log \delta}
$$

exists, then the other limit also exists, and, in this case,

$$
\operatorname{dim}_{\mathrm{B}}\left(E_{\theta, a}\right)+d\left(\nu^{\theta}, a\right)=s .
$$

Theorem 5. For every $\theta \in[0, \pi / 2)$ and for $\nu^{\theta}$-almost all $a \in I^{\theta}$ we have

$$
\operatorname{dim}_{\mathrm{B}}\left(E_{\theta, a}\right)=s-\operatorname{dim}_{\mathrm{H}}\left(\nu^{\theta}\right) \geq s-1 .
$$

The assertion includes that the box dimension exists.

2.3. The case of absolutely continuous $\nu^{\theta}$. It is well known (see 12, Theorem 9.7]) that $\nu^{\theta} \ll \mathcal{L}$ eb for Lebesgue almost all $\theta \in[0, \pi / 2)$.

Theorem 6. Suppose that $\theta$ satisfies $\nu^{\theta} \ll \mathcal{L}$ eb.

(a): For Leb-almost all $a \in I^{\theta}$, there exist $0<c_{3}(\theta, a)<c_{4}(\theta, a)<\infty$ such that

$$
\forall n, \quad c_{3}(\theta, a) \cdot\left(\frac{8}{3}\right)^{n}<N_{\theta, a}(n)<c_{4}(\theta, a) \cdot\left(\frac{8}{3}\right)^{n} .
$$

(b): In particular, for Lebesgue almost all $a \in I^{\theta}, \operatorname{dim}_{\mathrm{B}}\left(E_{\theta, a}\right)$ exists and is equal to $s-1$.

2.4. Behavior for rational slope. Recently Liu, Xi and Zhao proved

Theorem 7 ([1]). Let $\tan \theta \in \mathbb{Q}$. For Lebesgue almost all $a \in I^{\theta}$ we have

$$
\operatorname{dim}_{\mathrm{B}}\left(E_{\theta, a}\right)=\operatorname{dim}_{\mathrm{H}}\left(E_{\theta, a}\right)=d^{\theta}(\mathcal{L} \mathrm{eb}) \leq \frac{\log 8}{\log 3}-1,
$$

where $d^{\theta}(\mathcal{L}$ eb) is a constant depending only on $\theta$.

They conjectured the strict inequality.

If $\tan \theta \in \mathbb{Q}$, then the dimensions in Proposition 3 are equal.

Proposition 8. If $\tan \theta \in \mathbb{Q}$, then there is a constant $d^{\theta}\left(\nu^{\theta}\right)$ such that

$$
d^{\theta}\left(\nu^{\theta}\right):=d_{H}^{\theta}=d_{\underline{B}}^{\theta}=d_{\bar{B}}^{\theta} .
$$

Our main theorem asserts the strict inequality. 
Theorem 9. If $\tan \theta \in \mathbb{Q}$, then, for Lebesgue almost all $a \in I^{\theta}$, we have

$$
d^{\theta}(\mathcal{L} \mathrm{eb}):=\operatorname{dim}_{\mathrm{B}}\left(E_{\theta, a}\right)=\operatorname{dim}_{\mathrm{H}}\left(E_{\theta, a}\right)<\frac{\log 8}{\log 3}-1 .
$$

It now follows from Proposition 4 that the local dimension of the self-similar measure $\nu^{\theta}$ is Lebesgue almost everywhere equal to a constant which is greater than 1.

Corollary 10. If $\tan \theta \in \mathbb{Q}$, then, for Lebesgue almost all $a \in I^{\theta}$, we have

$$
d\left(\nu^{\theta}, a\right)=s-d^{\theta}(\mathcal{L} \mathrm{eb})>1 .
$$

Comparing (41) with (12) shows that, when $\tan \theta \in \mathbb{Q}$, the measure $\nu^{\theta}$ on $I^{\theta}$ is singular w.r.t. Lebesgue measure, in contrast to the absolute continuity in Section 2.3. It turns out that there are many slices which do not have the small dimension of Theorem 9 .

Theorem 11. If $\tan \theta \in \mathbb{Q}$, then, for $\nu^{\theta}$-almost all $a \in I^{\theta}$,

$$
\operatorname{dim}_{\mathrm{H}}\left(E_{\theta, a}\right)=s-\operatorname{dim}_{\mathrm{H}}\left(\nu^{\theta}\right) \geq s-1 .
$$

A result related to Corollary 10 was recently proved by Feng and Sidorov; see 5]. Their Proposition 1.4 says that if $\rho$ is the reciprocal of some Pisot number in $(1,2)$, the value taken at Lebesgue almost every point by the local dimension of the Bernoulli convolution $\mu_{\rho}$ is strictly greater than 1.

Organization of the paper: In Section 3 we develop our method of symbolic dynamics and prove Theorem 9 Then in Section 4 we prove the remaining results by using some notation and Proposition 18 from Section 3 .

\section{The PROOF OF OUR MAIN RESUlt}

We make the standing hypothesis that $\tan \theta=\frac{M}{N} \geq 0$, where the natural numbers $M, N$ are coprime.

If $3 \mid N$, then $3 \nmid M$ and, using the isometry $(x, y) \mapsto(y, x)$, we may consider $\tan \theta=N / M$ instead; thus we may assume that $3 \nmid N$. Because of the isometry $(x, y) \mapsto(1-x, 1-y)$ we do not need to consider $a \in\left[-\frac{M}{N}, 0\right)$.

The idea of the proof of Theorem 7 was as follows: The authors found three non-negative integer matrices $A_{0}, A_{1}, A_{2}$ such that corresponding to the first $n$ digits $\left(a_{1}, \ldots, a_{n}\right) \in\{0,1,2\}^{n}$ of the base 3 expansion of $a \in[0,1]$ they could approximate the minimal number of squares of size $1 / 3^{n}$ one needs to cover the $n$-th approximation of $F$ by the norm of $A_{a_{1}} \cdots A_{a_{n}}$.

Our method is similar but we use different matrices $A_{0}, A_{1}, A_{2}$, which carry more geometric meaning. In Proposition 12 the subadditive ergodic theorem is used to express Hausdorff dimension in terms of the average of the logarithm of the norm of products of $A_{0}, A_{1}, A_{2}$. In Proposition 18 we show that one of these products $B_{1}$ has each row either all zero or all positive (which requires extra care in Section 3.3 if $N$ is even). In Section 3.4 we consider the action of $A_{0}, A_{1}, A_{2}$ on the right on the simplex $\Delta$ of positive vectors as an Iterated Function System and, after studying powers of $B_{1}$, show that this IFS is contracting on average. In Section 3.5 our Theorem 9 is proved using the property that there is a positive measure subset of the invariant set of the IFS, where the various products do not all expand by the same amount, and a theorem of Furstenberg about the integral representation of the Lyapunov exponent of a random matrix product. 

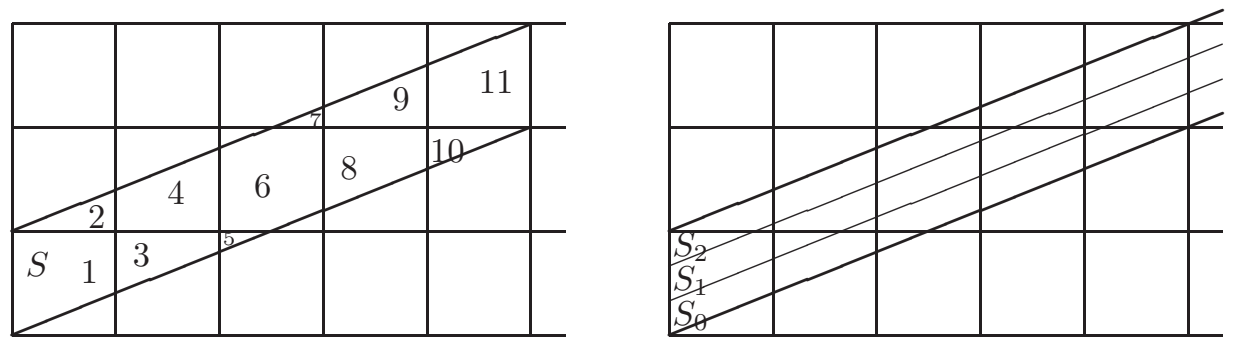

Figure 2. Tiling $S$ when $(N, M)=(5,2), K=11$

3.1. Our transition matrices. Now the interior of the strip

$$
S:=\left\{(x, y) \in \mathbb{R}^{2}: x \in[0, N], y-x \tan \theta \in[0,1]\right\}
$$

meets the $2 N+M-1\left(=K\right.$, say) unit squares $I^{2}+z$ for $z$ in

$$
\left\{\left(q,\left[q \frac{M}{N}\right]\right),\left(q,\left[q \frac{M}{N}\right]+1\right): 0 \leq q<N\right\} \cup\left\{\left(\left[r \frac{N}{M}\right], r+1\right): 0<r<M\right\} .
$$

In the tiling of $S$ by its intersection with these squares we number the tiles

$$
Q_{i}:=\left((q, r)+I^{2}\right) \cap S, 1 \leq i \leq K
$$

in increasing order of $q$ and, for given $q$, in increasing order of $r$. Let us denote the $(q, r)$ in (13) as $\left(q_{i}, r_{i}\right)$. That is,

$$
Q_{i}=\left(\left(q_{i}, r_{i}\right)+I^{2}\right) \cap S, \text { and } Q_{i} \cap \operatorname{int}(S) \neq \emptyset .
$$

Figure 2 illustrates the case $M / N=2 / 5, K=11$.

Consider the three parallel narrower infinite strips $S_{0}, S_{1}, S_{2}$ with

$$
S_{t}:=\left\{(x, y) \in \mathbb{R}^{2}: y-x \tan \theta-t / 3 \in[0,1 / 3]\right\}
$$

and the expanding maps

$$
\psi_{t}: S_{0} \cup S_{1} \cup S_{2} \rightarrow S_{t}, \quad \psi_{t}(x, y):=(x / 3,(y+t) / 3), \quad t=0,1,2 .
$$

Then

$$
E_{\theta, a}=F \cap \bigcap_{n=1}^{\infty} \psi_{a_{n-1}} \circ \cdots \circ \psi_{a_{1}} S_{a_{n}}
$$

expresses $E_{\theta, a}$ as the intersection with $F$ of strips of vertical height $3^{-n}$ chosen according to the expansion $0 . a_{1} a_{2} a_{3} \ldots$ of $a$ in base 3 .

Consider the intersection of $S_{t}$ with the eight squares of side $3^{-1}$ that cover $F$ and, correspondingly, of $3 S_{t}$ with $\left(q^{\prime}, r^{\prime}\right)+I^{2}$, where $q^{\prime}, r^{\prime}$ are not both congruent to 1 mod 3. We define, for $t \in\{0,1,2\}$, the $K \times K$ transition matrix $A_{t}$, with entries 0 and 1 so that its $(i, j)$-th entry is 1 if and only if $3\left(Q_{i} \cap S_{t}\right)$ contains $(\ell N, t+\ell M)+Q_{j}$ for some $\ell \in\{0,1,2\}$ with $q_{j}+\ell N, r_{j}+t+\ell M$ not both congruent to $1 \bmod 3$. That is,

$$
A_{t}(i, j)=1 \Leftrightarrow\left\{\begin{array}{c}
\exists \ell \in\{0,1,2\}, Q_{i} \cap S_{t} \supset 3^{-1}\left((\ell N, t+\ell M)+Q_{j}\right) \text { and } \\
\text { either } q_{j}+\ell N \neq 1 \bmod 3 \text { or } r_{j}+t+\ell M \not \equiv 1 \bmod 3
\end{array}\right\} .
$$




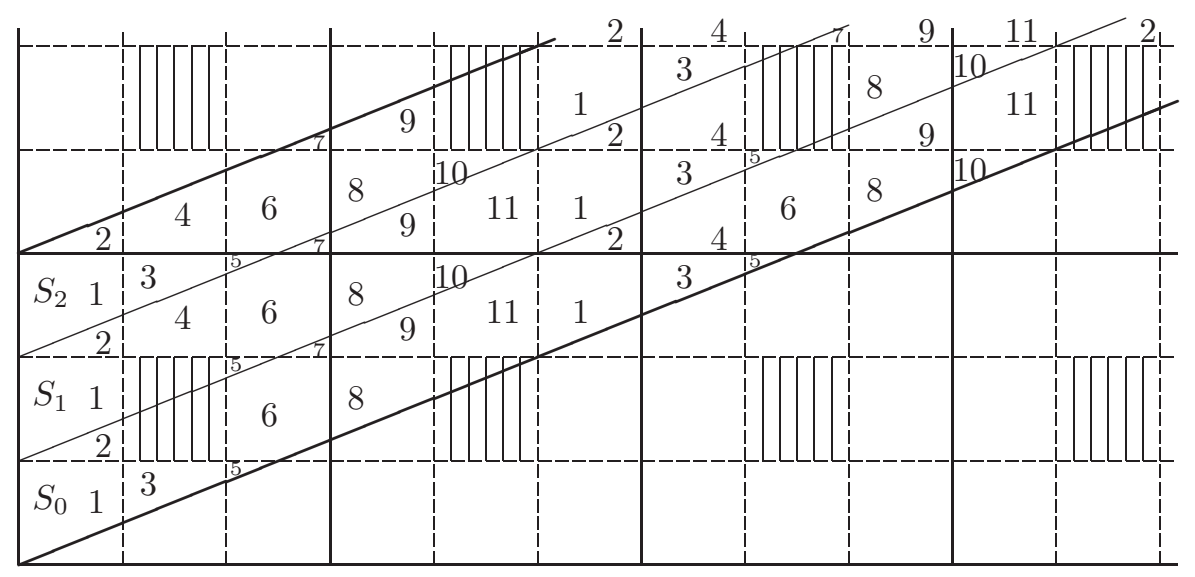

Figure 3. Tiling each strip $S_{t}$ when $(N, M)=(5,2)$

In Figure 3 the label $j$ is marked in $3^{-1}\left((\ell N, t+\ell M)+Q_{j}\right)$, and this illustrates, for example, that the first three rows of these transition matrices are

$$
\begin{aligned}
A_{0} & =\left(\begin{array}{lllllllllll}
1 & 1 & 1 & 0 & 1 & 1 & 1 & 0 & 0 & 0 & 0 \\
0 & 0 & 0 & 0 & 0 & 0 & 0 & 0 & 0 & 0 & 0 \\
1 & 0 & 0 & 0 & 0 & 0 & 0 & 1 & 1 & 0 & 1 \\
\cdots & & & & & & & & & &
\end{array}\right), \\
A_{1} & =\left(\begin{array}{ccccccccccc}
1 & 1 & 0 & 1 & 1 & 1 & 0 & 0 & 0 & 0 & 0 \\
0 & 0 & 0 & 0 & 0 & 0 & 1 & 0 & 0 & 0 & 0 \\
0 & 0 & 0 & 0 & 0 & 0 & 0 & 1 & 0 & 1 & 0 \\
\cdots & & & & & & & & &
\end{array}\right), \\
A_{2} & =\left(\begin{array}{lllllllllll}
1 & 0 & 1 & 0 & 1 & 0 & 0 & 0 & 0 & 0 & 0 \\
0 & 1 & 0 & 1 & 0 & 1 & 1 & 0 & 0 & 0 & 0 \\
0 & 0 & 0 & 0 & 0 & 0 & 0 & 0 & 0 & 0 & 0 \\
\cdots & & & & & & & & &
\end{array}\right) .
\end{aligned}
$$

The involution $(x, y) \mapsto(N, M+1)-(x, y)$ sends $S_{1}$ to itself, exchanges $S_{0}$ and $S_{2}$, and sends $Q_{i}$ to $Q_{K+1-i}$. Therefore these matrices exhibit the symmetries

$$
\begin{aligned}
\forall i, j \in\{1, \ldots, K\} \quad A_{0}(i, j) & =A_{2}(K+1-i, K+1-j), \\
A_{1}(i, j) & =A_{1}(K+1-i, K+1-j) .
\end{aligned}
$$

Consider transitions from $Q_{i}=\left((q, r)+I^{2}\right) \cap S$ to $Q_{j}=\left(\left(q^{\prime}, r^{\prime}\right)+I^{2}\right) \cap(S+(0, t))$. For the nine cases $\ell, t \in\{0,1,2\}, A_{t}(i, j)=1$ when $Q_{i}=\left(\left(\left[\left(q^{\prime}+\ell N\right) / 3\right],\left[\left(r^{\prime}+t+\right.\right.\right.\right.$ $\left.\ell M) / 3])+I^{2}\right) \cap S$ except for any case where

$$
q^{\prime}+\ell N \equiv r^{\prime}+t+\ell M \equiv 1 \bmod 3,
$$

which corresponds to a square deleted from $F$.

Because $3 \nmid N$, given $j$, (18) determines $(\ell, t)$, and so each of $A_{0}, A_{1}, A_{2}$ has each column sum equal to 2 or 3 and

$$
A_{s}:=A_{0}+A_{1}+A_{2} \text { has each column sum } 8 .
$$


If $\mathbf{a}_{0}, \mathbf{a}_{1}, \mathbf{a}_{2}$ and $\mathbf{1}$ denote the row vectors in $\mathbb{R}^{K}$ for which each entry of $\mathbf{1}$ is 1 and each $A_{\ell}$ has the row vector of its column sums equal to $3.1-\mathbf{a}_{t}$, then each entry of $\mathbf{a}_{t}$ is 0 or 1 and $\mathbf{a}_{0}+\mathbf{a}_{1}+\mathbf{a}_{2}=\mathbf{1}$.

Now $A_{t}(i, j)=1$ whenever $Q_{i} \cap S_{t}$ contains a $3^{-1}$-sized copy of $Q_{j}$, and $A_{u}(j, k)=$ 1 when $Q_{j} \cap S_{u}$ contains a $3^{-1}$-sized copy of $Q_{k}$, so the $(i, k)$-th entry of $A_{t} A_{u}$ is the number of the $8^{2}$ squares of side $3^{-2}$ covering $F$ that meet $Q_{i} \cap S_{t} \cap \psi_{t}\left(S_{u}\right)$ in a $3^{-2}$-sized copy of $Q_{k}$. By induction, the $(1, k)$-th entry in the product matrix $A_{a_{1}} A_{a_{2}} \ldots A_{a_{n}}$ is the number of the $8^{n}$ squares of side $3^{-n}$ covering $F$ that meet $Q_{1} \cap S_{a_{1}} \cap \psi_{a_{1}}\left(S_{a_{2}}\right) \cap \ldots \cap\left(\psi_{a_{1}} \circ \cdots \circ \psi_{a_{n-1}}\right)\left(S_{a_{n}}\right)$ in a $3^{-n}$-sized copy of $Q_{k}$. Thus the first row of $A_{a_{1}} A_{a_{2}} \ldots A_{a_{n}}$ counts the elements of a $\left(3^{-n} \sqrt{2}\right)$-cover of $E_{\theta, a}$. (In the cases $(N, M)=(1,1)$ and $(2,1)$, certain matrix entries are 2 , and the elements of the cover are still counted correctly.) Thus we get:

Proposition 12. For every $a \in[0,1], a=\sum_{i=1}^{\infty} a_{i} 3^{-i}$ we have

$$
\overline{\operatorname{dim}}_{\mathrm{B}} E_{\theta, a} \leq \frac{1}{\log 3} \limsup _{n \rightarrow \infty} \frac{1}{n} \log \left\|A_{a_{1} \ldots a_{n}}\right\|_{1},
$$

where $A_{a_{1} \ldots a_{n}}$ denotes $A_{a_{1}} \cdots A_{a_{n}}$ and $\|\cdot\|_{1}$ denotes the sum of the moduli of the entries. For almost all $a=\sum_{i=1}^{\infty} a_{i} \cdot 3^{-i}$ the right hand side gives the same value and we can replace limsup by lim. Consider the random product of the matrices $A_{0}, A_{1}, A_{2}$, each taken with probability $1 / 3$ independently in every step. Then the Lyapunov exponent $\gamma$ of this random matrix product is the almost sure value of the limit above. That is,

$$
\gamma:=\lim _{n \rightarrow \infty} \frac{1}{n} \log \left\|A_{a_{1} \ldots a_{n}}\right\|_{1}, \text { for a.a. }\left(a_{1}, a_{2}, \ldots\right) .
$$

Similarly,

$$
\gamma=\lim _{n \rightarrow \infty} \frac{1}{n} \sum_{i_{1} \ldots i_{n}} \frac{1}{3^{n}} \log \left\|A_{i_{1} \ldots i_{n}}\right\|_{1} .
$$

Further,

$$
\gamma \in[\log 2, \log 3]
$$

As an easy consequence of Theorem 7 the definition of $\gamma$ and the translation invariance of the Lebesgue measure, we obtain the following proposition.

Proposition 13. If $\tan \theta \in \mathbb{Q}$, then for Lebesgue almost all $a \in I^{\theta}$ we have

$$
\operatorname{dim}_{\mathrm{B}}\left(E_{\theta, a}\right)=\operatorname{dim}_{\mathrm{H}}\left(E_{\theta, a}\right)=\frac{\gamma}{\log 3} .
$$

We will use the following definitions.

Definition 14. The symbolic space to code the translation parameter $a \in[0,1]$ is $\Sigma:=\{0,1,2\}^{\mathbb{N}}$. Let $\pi_{y}: \Sigma \rightarrow[0,1]$ be defined by

$$
\pi_{y}(\mathbf{i}):=\sum_{k=1}^{\infty} i_{k} \cdot 3^{-k}, \quad \mathbf{i}=\left(i_{1}, i_{2}, \ldots\right) .
$$

We denote the uniform distribution on $\Sigma$ by $\mathbb{P}:=\left\{\frac{1}{3}, \frac{1}{3}, \frac{1}{3}\right\}^{\mathbb{N}}$. The push down measure $\left(\pi_{y}\right)_{*}$ of $\mathbb{P}$ by $\pi_{y}$ is the Lebesgue measure $\mathcal{L}$ eb on $[0,1]$. The measure $\mathbb{P}$ is ergodic with respect to $(\Sigma, \sigma)$, where $\sigma$ is the left shift on $\Sigma$. 
As an easy case first consider the intersection of $F$ with horizontal lines.

Proposition 15. For almost every $a, \operatorname{dim}_{\mathrm{H}}\left(E_{0, a}\right)=\frac{1}{3} \log 18 / \log 3<\log 8 / \log 3-$ 1.

Proof. If $\tan \theta=0 / 1$, then the above construction gives $K=2 N+M-1=1$ and $1 \times 1$ matrices $A_{0}=A_{2}=(3), A_{1}=(2)$. Then $E_{0, a}$ is covered by $A_{a_{1}} \ldots A_{a_{n}}$ squares of side $3^{-n}$. This leads us to study the function on $\Sigma:=\{0,1,2\}^{\mathbb{N}}$ taking the value $\log 2$ where the first symbol is 1 and $\log 3$ elsewhere, whose integral with respect to $\mathbb{P}$ is $\frac{1}{3} \log 18$.

So, from now on we may assume that $M / N \neq 0$. By symmetry, without loss of generality we may assume that $M / N>0$.

To prove Proposition 12 we need the following simple observation, which will also be used later.

Fact 16. Consider the non-negative $K \times K$ matrices $A, B$. Let

$$
\mathbf{c}_{A}(j):=\sum_{i=1}^{K} A(i, j) \text { and } \mathbf{r}_{B}(i):=\sum_{j=1}^{K} B(i, j)
$$

be the $j$-th column sum and the $i$-th row sum of the matrices $A, B$ respectively. Then

$$
\|A \cdot B\|_{1}=\sum_{i} \mathbf{c}_{A}(i) \cdot \mathbf{r}_{B}(i)
$$

Proof. The proof of Fact 16 is a simple calculation.

Now we turn to the proof of Proposition 12

Proof of Proposition 12. The inequality in (20) immediately follows from the discussion right above the proposition. The fact that we can replace lim sup by lim in (20) is an immediate corollary of the sub-additive ergodic theorem (see [18, p. 231]). For non-negative matrices it is easy to check that $\|\cdot\|_{1}$ is submultiplicative. Indeed

$$
\|A \cdot B\|_{1}=\sum_{i} \mathbf{c}_{A}(i) \cdot \mathbf{r}_{B}(i) \leq \sum_{i=1}^{K} \sum_{j=1}^{K} \mathbf{c}_{A}(i) \cdot \mathbf{r}_{B}(j)=\|A\|_{1} \cdot\|B\|_{1} .
$$

This implies that the sequence of the bounded functions $f_{k}: \Sigma \rightarrow \mathbb{R}$,

$$
f_{k}(\mathbf{i}):=\log \left\|A_{i_{k}}^{T} \cdots A_{i_{1}}^{T}\right\|_{1}=\log \left\|A_{i_{1} \ldots i_{k}}\right\|_{1},
$$

is sub-additive. Using the ergodicity of $\mathbb{P}$ and the sub-additive ergodic theorem [18, p. 231] we obtain that the limit

$$
\gamma:=\lim _{n \rightarrow \infty} \frac{1}{n} \cdot f_{n}(\mathbf{i})=\lim _{n \rightarrow \infty} \frac{1}{n} \cdot \log \left\|A_{i_{1}} \cdots A_{i_{n}}\right\|_{1}
$$

exists for $\mathbb{P}$-almost all $\left(a_{1}, a_{2}, \ldots\right) \in \Sigma$ and gives the same value. Further, using

$$
\frac{1}{n} \int f_{n}(\mathbf{i}) d \mathbb{P}(\mathbf{i})=\frac{1}{n} \sum_{i_{1} \ldots i_{n}} \frac{1}{3^{n}} \log \left\|A_{i_{1} \ldots i_{n}}\right\|_{1},
$$

the sub-additive ergodic theorem implies that (22) holds. 
To verify (23) we make the following observation: each factor in the matrix product $A_{i_{1}} \cdots A_{i_{n}}$ has each column sum either 2 or 3 . This implies that all the column sums of the product matrix are between $2^{n}$ and $3^{n}$. This yields

$$
\forall\left[i_{1}, \ldots, i_{n}\right] \text { we have: } K \cdot 2^{n} \leq\left\|A_{i_{1}} \cdots A_{i_{n}}\right\|_{1} \leq K \cdot 3^{n} .
$$

The inequality (30) below is an immediate corollary of (20) and (21).

Corollary 17. The following holds:

$$
\overline{\operatorname{dim}}_{\mathrm{B}} E_{\theta, a} \leq \frac{\gamma}{\log 3} .
$$

3.2. Positive rows in some products of our matrices. Recall from Section 1 that

$$
3 \nmid N .
$$

In this section and the next we prove

Proposition 18. There exists $n_{0}$ and $\left(a_{1} \ldots a_{n_{0}}\right) \in\{0,1,2\}^{n_{0}}$ such that the rows of the matrix $A_{a_{1} \ldots a_{n_{0}}}$ are vectors with either all positive or all zero elements.

Our approach is as follows. A row will be positive when labels 1 to $K$ appear in the intersection of each square with the strip in the $n_{0}$-th version of Figure 3. The labelling depends on the relation between $N$ and 3 . When $M / N<1$ we shall find the labels near the left or right edge of the square in positions corresponding to squares in the $n_{0}$-th stage in the construction of $F$. When $M / N \geq 1$ the strip may not be near either edge, so we shall search instead in small rectangles with diagonal of slope near $M / N$ and carefully chosen horizontal and vertical coordinates.

3.2.1. An IFS leaving $S$ invariant. First we introduce some notation. For $t, \ell \in$ $\{0,1,2\}$ we define the contraction:

$$
\psi_{t}^{\ell}(x, y):=\frac{1}{3} \cdot[x+\ell \cdot N, y+\ell \cdot M+t] .
$$

To compute the $n$-fold compositions we introduce the following notation:

$$
\ell_{1, n}:=\ell_{1} 3^{n-1}+\cdots+\ell_{n-1} \cdot 3+\ell_{n}
$$

and

$$
a_{1, n}:=a_{1} 3^{n-1}+\cdots+a_{n-1} \cdot 3+a_{n} .
$$

If we write $\psi_{a_{1} \ldots a_{n}}^{\ell_{1} \ldots \ell_{n}}$ for $\psi_{a_{1}}^{\ell_{1}} \circ \cdots \circ \psi_{a_{n}}^{\ell_{n}}$ and $S_{a_{1} \ldots a_{n}}^{\ell_{1} \ldots \ell_{n}}$ for $\psi_{a_{1} \ldots a_{n}}^{\ell_{1} \ldots \ell_{n}}(S)$, then we have

$$
\psi_{a_{1} \ldots a_{n}}^{\ell_{1} \ldots \ell_{n}}(x, y)=\frac{1}{3^{n}} \cdot\left[x+N \cdot \ell_{1, n}, y+M \cdot \ell_{1, n}+a_{1, n}\right]
$$

and

$$
S=\bigcup_{\left(a_{1} \ldots a_{n}\right),\left(\ell_{1} \ldots \ell_{n}\right) \in\{0,1,2\}^{n}} S_{a_{1} \ldots a_{n}}^{\ell_{1} \ldots \ell_{n}},
$$

where the sets on the right hand side have disjoint interior. The set $S_{a_{1} \ldots a_{n}}^{\ell_{1} \ldots \ell_{n}}$ is the intersection of the slope $\theta$ level $n$ strip

$$
S_{a_{1} \ldots a_{n}}:=\left\{(x, y): 0 \leq x \leq N \text { and } 0 \leq y-\left(x \cdot \tan \theta+\frac{a_{1}}{3}+\cdots+\frac{a_{n}}{3^{n}}\right) \leq \frac{1}{3^{n}}\right\},
$$


with the level $n$ vertical strip

$$
V^{\ell_{1} \ldots \ell_{n}}:=\left\{(x, y): N \cdot\left(\frac{\ell_{1}}{3}+\cdots+\frac{\ell_{n}}{3^{n}}\right) \leq x<N \cdot\left(\frac{\ell_{1}}{3}+\cdots+\frac{\ell_{n}}{3^{n}}+\frac{1}{3^{n}}\right)\right\},
$$

for all $\left(\ell_{1} \ldots \ell_{n}\right),\left(a_{1} \ldots a_{n}\right) \in\{0,1,2\}^{n}$. See Figure 4 .

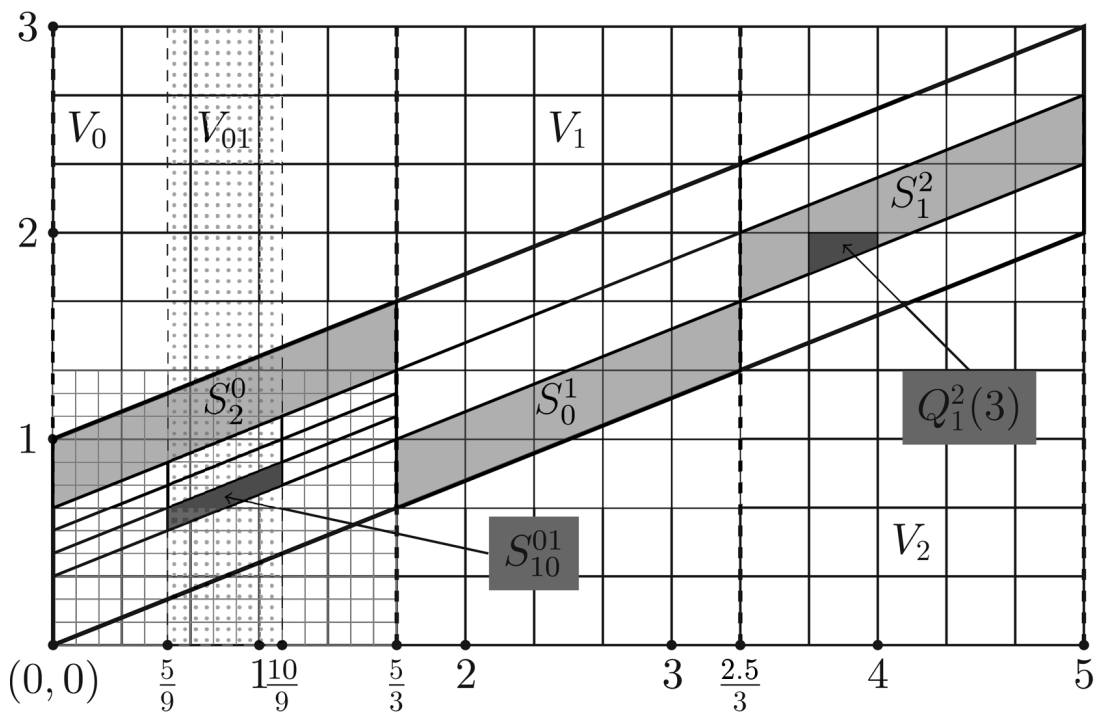

Figure 4. The subsets defined in (35) and (36) when $M / N=2 / 5$.

Earlier in (14) we defined the $K=2 N+M-1$ different level zero squares which together cover $S$. Similarly, here we define the level $n$ square of shape $j$ in $S_{a_{1} \ldots a_{n}}^{\ell_{1} \ldots \ell_{n}}$ by

$$
Q_{a_{1} \ldots a_{n}}^{\ell_{1} \ldots \ell_{n}}(j):=\psi_{a_{1} \ldots a_{n}}^{\ell_{1} \ldots \ell_{n}}\left(Q_{j}\right) .
$$

\subsubsection{The $n$-th approximation of the translated copies of the Sierpinski carpet.}

Definition 19. Let $\widetilde{F}$ be the union of the translated copies of the Sierpinski carpet to those unit squares that intersect $S$. That is,

$$
\widetilde{F}:=\bigcup_{i=1}^{K}\left(\left(q_{i}, r_{i}\right)+F\right) .
$$

Let $\widetilde{F}^{n}$ be the level $n$ approximation of $\widetilde{F}$. Put

$$
\begin{aligned}
& U_{i}^{n}=\left\{u \in\left[q_{i}, q_{i}+1\right): \exists\left(u_{1}, \ldots, u_{n}\right) \in\{0,1,2\}^{m} \text { s.t. } u=q_{i}+\sum_{m=1}^{n} u_{m} \cdot 3^{-m}\right\}, \\
& V_{i}^{n}=\left\{v \in\left[r_{i}, r_{i}+1\right): \exists\left(v_{1}, \ldots, v_{n}\right) \in\{0,1,2\}^{m} \text { s.t. } v=r_{i}+\sum_{m=1}^{n} u_{m} \cdot 3^{-m}\right\}
\end{aligned}
$$

and say that $u_{m}, v_{m}$ are the $m$-th ternary (that is, base 3 ) digits of $u, v$ respectively. For some $n_{1} \geq n_{2}$ and for $u \in U_{i}^{n_{1}}$ and $v \in V_{i}^{n_{2}}$ we define the so-called level $\left(n_{1}, n_{2}\right)$ 
grid rectangle in the square $\left(q_{i}, r_{i}\right)+I^{2}$ :

$$
R_{i}(u, v):=\left(q_{i}, r_{i}\right)+\left(\sum_{m=1}^{n_{1}} u_{m} \cdot 3^{-m}, \sum_{m=1}^{n_{2}} v_{m} \cdot 3^{-m}\right)+\left[0,3^{-n_{1}}\right] \times\left[0,3^{-n_{2}}\right] .
$$

The collection of all level $\left(n_{1}, n_{2}\right)$ grid rectangles in $\left(q_{i}, r_{i}\right)+I^{2}$ is called $\mathcal{R}_{i}\left(n_{1}, n_{2}\right)$. That is,

$$
\mathcal{R}_{i}\left(n_{1}, n_{2}\right):=\left\{R_{i}(u, v): u \in U_{i}^{n_{1}} \text { and } v \in V_{i}^{n_{2}}\right\} .
$$

When $n_{1}=n_{2}$, then the elements of

$$
\mathcal{C}_{i}(n):=\mathcal{R}_{i}\left(n_{1}, n_{2}\right), \quad n=n_{1}=n_{2}
$$

are called level $n$ grid squares in $\left(q_{i}, r_{i}\right)+I^{2}$. Those level $n$ grid squares that are contained in $\widetilde{F}^{n}$ are called $n$-cylinder squares.

For a given level $n$ grid square we can decide if it is an $n$-cylinder square using the following fact, whose proof follows immediately from the observation that all the elements $(x, y)$ of the Sierpinski carpet $F$ can be represented as $(x, y)=\sum_{k=1}^{\infty}\left(\frac{u_{k}}{3^{k}}, \frac{v_{k}}{3^{k}}\right)$ such that for all $k$ either $u_{k} \in\{0,2\}$ or $v_{k} \in\{0,2\}$.

Fact 20. The level $n$ grid square $R_{i}(u, v) \in \mathcal{C}_{i}(n)$ with $u=q_{i}+\sum_{m=1}^{n} u_{m} \cdot 3^{-m}$ and $v=r_{i}+\sum_{m=1}^{n} v_{m} \cdot 3^{-m}$ is an $n$-cylinder square if and only if

$$
\forall 1 \leq p \leq n, \quad \text { either } u_{p} \in\{0,2\} \text { or } v_{p} \in\{0,2\} .
$$

Fact 21. If $Q_{a_{1} \ldots a_{n}}^{\ell_{1} \ldots \ell_{n}}(j) \subset\left(q_{i}, r_{i}\right)+I^{2}$, then the inclusion

$$
Q_{a_{1} \ldots a_{n}}^{\ell_{1} \ldots \ell_{n}}(j) \subset \widetilde{F}^{n} \cap\left(\left(q_{i}, r_{i}\right)+I^{2}\right)
$$

is equivalent to the following assertion:

(Assertion): Let $\left(u_{1}, \ldots, u_{n}\right),\left(v_{1}, \ldots, v_{n}\right) \in\{0,1,2\}^{n}$ be defined by

$$
\psi_{a_{1} \ldots a_{n}}^{\ell_{1} \ldots \ell_{n}}\left(q_{j}, r_{j}\right)=\left(q_{i}, r_{i}\right)+\frac{1}{3^{n}}\left(\sum_{\ell=1}^{n} 3^{n-\ell} \cdot u_{\ell}, \sum_{\ell=1}^{n} 3^{n-\ell} \cdot v_{\ell}\right) .
$$

Then for every $1 \leq p \leq n$ we have

$$
\text { either } u_{p} \in\{0,2\} \text { or } v_{p} \in\{0,2\} \text {. }
$$

Lemma 22. There is $m_{0}$ such that $\left\{3^{m_{0}}+3^{2 m_{0}} \cdots+3^{k m_{0}}\right\}_{k=1}^{N}$ is a full residue system modulo $N$.

Proof. Clearly we can find $k<\ell$ such that $3^{k} \equiv 3^{\ell} \bmod N$. Let $m_{0}:=\ell-k$. Then

$$
3^{m_{0}} \equiv 1 \quad \bmod N
$$

holds (since we assumed that $3 \nmid N$ ). Thus $3^{m_{0}}+3^{2 m_{0}}+\cdots+3^{k m_{0}} \equiv k \bmod N$.

Definition 23. (a): First we define $k_{0}$ as the smallest non-negative integer satisfying $M / N<3^{k_{0}}$. That is, if $M / N \geq 1$, then

$$
3^{k_{0}-1} \leq M / N<3^{k_{0}} .
$$

On the other hand if $M / N<1$, then $k_{0}:=0$.

(b): We fix $m_{0}$ which satisfies (42). 
(c): Finally, we introduce the equivalence relation $\sim$ on $\{0, \ldots, N-1\}$ as follows:

if $N$ is odd: then $k \sim \ell$ holds for all $k, \ell$;

if $N$ is even: then $k \sim \ell$ holds iff either both $k$ and $\ell$ are even or both of them are odd.

(d): Assume that $M / N<1$. We shall argue later in proving Proposition 8 that for all shapes $Q_{i}, 1 \leq i \leq K$, the region in $Q_{i}$ with first coordinate in the interval

$J_{0}^{n}(i)=\left[q_{i}, q_{i}+\left(3^{m_{0} \cdot N+1}\right) \cdot 3^{-n}\right)$ or $J_{2}^{n}(i)=\left[q_{i}+1-\left(3^{m_{0} \cdot N+1}+1\right) \cdot 3^{-n}, q_{i}+1-3^{-n}\right)$ contains an image of $Q_{j}$ by $\psi_{a_{1} \ldots a_{n}}^{\ell_{1} \ldots \ell_{n}}$ for an appropriately chosen $\ell_{1} \ldots \ell_{n}$.

The definition of the intervals in (44) will be much more complicated when $M / N \geq 1$.

3.2.3. The definition of the intervals $J_{0}^{n}(i), J_{2}^{n}(i)$ in the general case. First we have to place some restrictions on $\left(a_{1}, \ldots, a_{n}\right) \in\{0,1,2\}^{n}$ for the strip $S_{a_{1}, \ldots, a_{n}}$ considered. To do that we divide the set $\{1,2, \ldots, n\}$ into four regions:

$$
\mathcal{I}_{1}:=\left\{1, \ldots, 2 k_{0}\right\}, \quad \mathcal{I}_{2}:=\left\{2 k_{0}+1, \ldots, n_{*}\right\},
$$

where $n_{*}:=n-\left(m_{0} N+1\right)-4 N 3^{2 k_{0}}$ and

$$
\mathcal{I}_{3}:=\left\{n_{*}+1, \ldots, n^{*}\right\}, \quad \mathcal{I}_{4}:=\left\{n^{*}+1, \ldots, n\right\},
$$

where $n^{*}:=n_{*}+4 N 3^{2 k_{0}}=n-\left(m_{0} N+1\right)$. When $M / N<1$, then $k_{0}=0$ and in that case $\mathcal{I}_{1}=\emptyset$.

\section{Assumption (A1):}

(a): $a_{n}=0$

(b): $\forall i, \forall u \in U_{i}^{2 k_{0}}, v \in V_{i}^{k_{0}}$ we assume that both the top left and bottom right corners of the rectangle $R_{i}(u, v)$ are farther from $S_{a_{1} \ldots a_{n}}$ than $3^{-n_{*}}$. This can be arranged by excluding $N M 3^{3 k_{0}} \ll 3^{n_{*}}$ grid intervals of level $n_{*}-$ $\left(k_{0}+2\right)$ when selecting the level $n$ grid interval determined by $\left(a_{1}, \ldots, a_{n}\right)$ from the interval $[0,1]$. So, this is a restriction implemented by excluding some intervals whose indices are from $\mathcal{I}_{1} \cup \mathcal{I}_{2}$ and their total length is less than $N M 3^{3 k_{0}} \cdot 2 \cdot 3^{-n_{*}} \cdot 3^{k_{0}+2}$, so now we assume that $n$ is large enough that this is $\ll 1$.

(c): Let us denote the bottom edge of $S_{a_{1} \ldots a_{n}}$ by Bottom Ba $_{1} \ldots a_{n}$. See Figure 5. We define $y^{\alpha}\left(\bar{y}^{\alpha}\right)$ for $1 \leq \alpha \leq N 3^{2 k_{0}}\left(0 \leq \alpha \leq N 3^{2 k_{0}}-1\right)$ as the second coordinate of the intersection of the line Bottom Ba $_{1} \ldots a_{n}$ with the vertical line $\underline{x}^{\alpha}:=\alpha \cdot 3^{-2 k_{0}}-2 \cdot 3^{-\left(n_{*}+4 \alpha-1\right)}\left(\bar{x}^{\alpha}:=\alpha \cdot 3^{-2 k_{0}}+3^{-\left(n_{*}+4 \alpha+1\right)}\right)$ respectively. Note that all these $\underline{x}^{\alpha}, \bar{x}^{\alpha}$ lie in $[0, N]$. We assume that $\left(a_{1}, \ldots, a_{n}\right)$ is chosen in such a way that

c1: for all $1 \leq \alpha \leq N 3^{2 k_{0}}$, both the $\left(n_{*}+4 \alpha-1\right)$-th and the $\left(n_{*}+4 \alpha\right)$-th digits of the ternary expansion of $y^{\alpha}$ are zero and

c2: for all $0 \leq \alpha \leq N 3^{2 k_{0}}-1$, both the $\left(n_{*}+4 \alpha+1\right)$-th and $\left(n_{*}+4 \alpha+2\right)$-th digits of the ternary expansion of $\bar{y}^{\alpha}$ are zero.

Now we prove that there is a positive proportion (independent of $n$ ) of all possible $\left(a_{1}, \ldots, a_{n}\right) \in\{0,1,2\}^{n}$ for which assumptions (c1) and (c2) hold. First, for having 


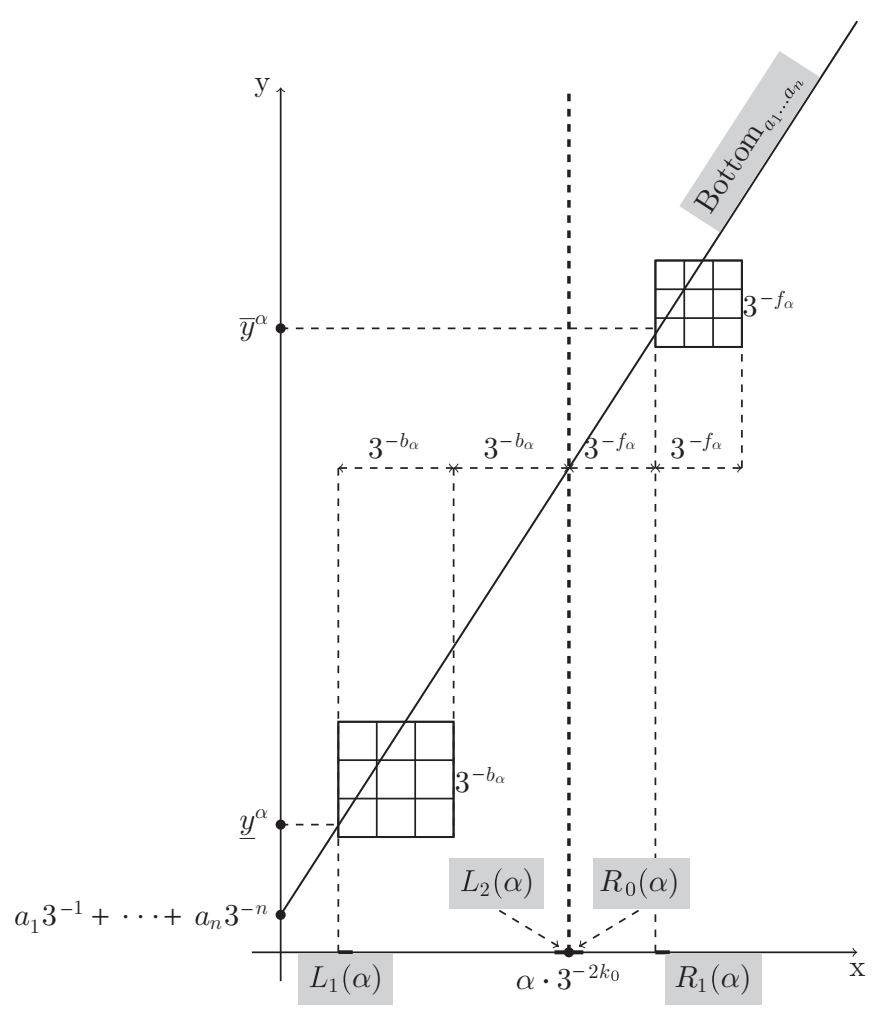

FiguRE $5 . L_{1}(\alpha), L_{2}(\alpha), R_{0}(\alpha), R_{1}(\alpha)$ are level $n^{*} \gg 2 k_{0}$ grid intervals.

a more convenient notation we write

$$
b_{\alpha}:=n_{*}+4 \alpha-1,1 \leq \alpha \leq N 3^{2 k_{0}} \text { and } f_{\alpha}:=n_{*}+4 \alpha+1,0 \leq \alpha \leq N 3^{2 k_{0}}-1 \text {. }
$$

(Here $f_{\alpha}$ refers to forward and $b_{\alpha}$ refers to backward relative to $\alpha 3^{-2 k_{0}}$; see Figure 5.)

Fact 24. There are $3^{n_{*}}$ possible choices of $\left(a_{1}, \ldots, a_{n}\right) \in\{0,1,2\}^{n}$ such that the following holds:

$$
\begin{array}{ll}
0=\underline{y}_{b_{\alpha}}^{\alpha}=\underline{y}_{b_{\alpha}+1}^{\alpha}, & \forall \alpha \in\left\{1, \ldots, N 3^{2 k_{0}}\right\} \text { and } \\
0=\bar{y}_{f_{\alpha}}^{\alpha}=\bar{y}_{f_{\alpha}+1}^{\alpha}, & \forall \alpha \in\left\{0, \ldots, N 3^{2 k_{0}}-1\right\},
\end{array}
$$

where $\underline{y}_{k}^{\alpha}\left(\bar{y}_{k}^{\alpha}\right)$ is the $k$-th ternary digit of $\underline{y}^{\alpha}\left(\bar{y}^{\alpha}\right)$ respectively.

Proof. Observe that

$$
\underline{y}^{\alpha}=\sum_{k=1}^{n} a_{k} 3^{-k}+\underline{z}^{\alpha} \text { and } \bar{y}^{\alpha}=\sum_{k=1}^{n} a_{k} 3^{-k}+\bar{z}^{\alpha},
$$

where

$$
\underline{z}^{\alpha}:=\frac{M}{N} \cdot \underline{x}^{\alpha} \text { and } \bar{z}^{\alpha}:=\frac{M}{N} \cdot \bar{x}^{\alpha}
$$

We prove that there is a way to choose the elements $a_{k} \in\{0,1,2\}$, for $k \in \mathcal{I}_{3} \cup \mathcal{I}_{4}$ such that (45) holds for all possible choices of $a_{k}, k \in \mathcal{I}_{1} \cup \mathcal{I}_{2}$. We construct these 
values $a_{k}$ for $k \in \mathcal{I}_{3} \cup \mathcal{I}_{4}$ by mathematical induction starting from $k=n$ and moving towards smaller values of $k$. Namely, we define $a_{k}:=0$ for all $k \in \mathcal{I}_{4}$. Fix an arbitrary $k^{\prime} \in \mathcal{I}_{3}$. We assume that we have already defined $a_{k}$ for all $k^{\prime} \leq k \leq n$. Clearly, we can either find a $1 \leq \alpha^{\prime} \leq N 3^{2 k_{0}}$ such that $k^{\prime} \in\left\{b_{\alpha^{\prime}}, b_{\alpha^{\prime}}+1\right\}$ or we can find a $0 \leq \alpha^{\prime} \leq N 3^{2 k_{0}}-1$ such that $k^{\prime} \in\left\{f_{\alpha^{\prime}}, f_{\alpha^{\prime}}+1\right\}$. For symmetry without loss of generality we may assume that we are in the latter case and $k^{\prime}=f_{\alpha^{\prime}}+1$. Then we compute the overflow $o_{k^{\prime}}$ from the $\left(k^{\prime}+1\right)$-th ternary place to the $k^{\prime}$ th ternary place when adding up $\sum_{k=k^{\prime}+1}^{n} a_{k} \cdot 3^{-k}$ and $\sum_{k=k^{\prime}+1}^{\infty} \bar{z}_{k}^{\alpha^{\prime}} \cdot 3^{-k}$. That is, if $\sum_{k=k^{\prime}+1}^{n} a_{k} \cdot 3^{-k}+\sum_{k=k^{\prime}+1}^{\infty} \bar{z}_{k}^{\alpha^{\prime}} \cdot 3^{-k}>3^{-k^{\prime}}$, then there is an overflow to the $k^{\prime}$-th ternary place and then $o_{k^{\prime}}:=1$; otherwise there is no overflow and $o_{k^{\prime}}:=0$. Observe that the value of $o_{k^{\prime}}$ depends only on the ternary digits $a_{k}$ for $k^{\prime}<k \leq n$ (which have been determined at this stage of the mathematical induction) and $z_{k}^{\alpha^{\prime}}$ for $k^{\prime}<k$ which are given numbers. So, we can compute the number $o_{k^{\prime}}$. It follows from (46) that $\bar{y}_{k^{\prime}}^{\alpha}=a_{k^{\prime}}+\bar{z}_{k^{\prime}}^{\alpha}+o_{k^{\prime}}$. Then for $a_{k^{\prime}}:=-\left(\bar{z}_{k^{\prime}}^{\alpha}+o_{k^{\prime}}\right) \bmod 3$ we obtain that $\bar{y}_{k^{\prime}}^{\alpha}=0$. We continue this process with doing the same first for $k^{\prime}-1$, then $k^{\prime}-2$, and so on for all $k \geq n_{*}+1$.

Fact 25. Let $J \subset[0,1]$ be a non-empty interval. Whenever $n$ is big enough we can choose $\left(a_{1}, \ldots, a_{n}\right) \in\{0,1,2\}^{n}$ which satisfies the requirements of assumption (A1) and

$$
\left[\sum_{k=1}^{n} a_{k} 3^{-k}, \sum_{k=1}^{n} a_{k} 3^{-k}+3^{-n}\right] \subset J .
$$

Proof. The three parts of the assumption posed restrictions for indices in different regions, so these restrictions cannot conflict. Let $G$ be the biggest grid interval, say level $g$, which is contained in $J$. This means that we need to fix the first $g$ ternary digits. In parts (a) and (c) of assumption (A1), we fixed the last $4 N 3^{2 k_{0}}+m_{0} N+1$ ternary digits of $\left(a_{1}, \ldots, a_{n}\right)$. So, from now on, we need to fix $g+4 N 3^{2 k_{0}}+m_{0} N+1$ ternary digits to provide that (47) and parts (a), (c) of assumption (A1) hold. In this way we restrict ourselves to a set of level $n$ grid intervals with a total length of at least $3^{-\left(g+4 N 3^{2 k_{0}}+m_{0} N+1\right)}$ (which does not depend on $n$ ) among which only an amount of total length of $N M 3^{3 k_{0}} \cdot 3^{-\left(n_{*}-\left(k_{0}+2\right)\right)}$ (which tends to zero as $n \rightarrow \infty$ ) is lost for part (b). So, if $n$ is big enough, then we find an $\left(a_{1}, \ldots, a_{n}\right)$ satisfying the assumptions (A1) and (47).

The reason for part (c) of assumption (A1) is as follows:

Remark 26. We consider $\alpha \cdot 3^{-2 k_{0}}$ as the end point of two level $2 k_{0}$ grid intervals:

$$
I_{L}(\alpha):=\left[(\alpha-1) 3^{-2 k_{0}}, \alpha 3^{-2 k_{0}}\right] \text { and } I_{R}:=\left[\alpha 3^{-2 k_{0}},(\alpha+1) 3^{-2 k_{0}}\right] .
$$

Assume that the corresponding ternary digits of these intervals are $\left(u_{1}^{L}, \ldots, u_{2 k_{0}}^{L}\right)$ and $\left(u_{1}^{R}, \ldots, u_{2 k_{0}}^{R}\right)$. Let

$$
n_{\alpha, L}:=\#\left\{1 \leq \ell \leq 2 k_{0}: u_{\ell}^{L}=1\right\}, n_{\alpha, R}:=\#\left\{1 \leq \ell \leq 2 k_{0}: u_{\ell}^{R}=1\right\} .
$$

Then we define the level $n^{*}$ grid intervals

(50) $J_{L}(\alpha):=\left\{\begin{array}{ll}L_{1}(\alpha), & \text { if } n_{\alpha, L} \text { is odd } \\ L_{2}(\alpha), & \text { otherwise, }\end{array} J_{R}(\alpha):= \begin{cases}R_{1}(\alpha), & \text { if } n_{\alpha, L} \text { is odd, } \\ R_{0}(\alpha), & \text { otherwise, }\end{cases}\right.$ 
where the level $n^{*}$ grid intervals $L_{1}(\alpha), L_{2}(\alpha), R_{0}(\alpha), R_{1}(\alpha)$ are defined in Figure 5. In this way, $J_{L}(\alpha)$ and $J_{R}(\alpha)$ are level $n^{*}$ grid intervals contained in $I_{L}(\alpha)$ and $I_{R}(\alpha)$ respectively. For $V \in\{L, R\}$ we obtain the ternary digits of $J_{V}(\alpha)$ as the concatenation of $\left(u_{1}^{V}, \ldots, u_{2 k_{0}}^{V}\right)$ and a vector of $n^{*}-2 k_{0}$ components of all zeros or twos if $n_{\alpha, V}$ is an even number. If $n_{\alpha, V}$ is an odd number, then the ternary digits of $J_{V}(\alpha)$ are obtained in the same way with the difference that we have digit one in the $b_{\alpha}$-th place $\left(f_{\alpha}\right.$-th place) if $V=L(V=R)$ respectively. In this way for both $J_{L}(\alpha)$ and $J_{R}(\alpha)$ the number of ones among the ternary digits is an even number.

Definition 27. We say that $S_{a_{1} \ldots a_{n}}$ is an n-good strip if $\left(a_{1}, \ldots, a_{n}\right)$ satisfies assumption (A1).

From now on we fix $n$ and an $n$-good strip $S_{a_{1} \ldots a_{n}}$. For this strip $Q_{i}=\left(\left(q_{i}, r_{i}\right)+\right.$ $\left.I^{2}\right) \cap S$ is a relevant shape if $\operatorname{int}\left(S_{a_{1} \ldots a_{n}} \cap Q_{i}\right) \neq \emptyset$.

We remark that, in the case when $M / N<1$, we do not use part (c) of assumption (A1).

We recall that in Definition 23 we have already defined the intervals $J_{0}^{n}(i)=$ $\left[q_{i}, q_{i}+3^{-\left(n^{*}-1\right)}\right)$ and $\left[q_{i}+1-3^{-\left(n^{*}-1\right)}-3^{-n}, q+1-3^{-n}\right)$. Now we extend this definition to the case when $M / N \geq 1$ and $Q_{i}$ is a relevant shape for the strip $S_{a_{1} \ldots a_{n}}$. The idea of the construction is as follows: Using the notation of Definition 19 for every $1 \leq i \leq K$ we shall define, by an inductive procedure, $R_{i}\left(u^{k_{0}+\ell}, v^{\ell}\right)$, for $0 \leq \ell \leq k_{0}$ such that:

(C1): $S_{a_{1} \ldots a_{n}} \cap R_{i}\left(u^{k_{0}+\ell}, v^{\ell}\right) \neq \emptyset$.

(C2): $u^{k_{0}+\ell} \in U_{i}^{k_{0}+\ell}$ and $v^{\ell} \in V_{i}^{\ell}$ with

$$
u^{k_{0}+\ell}=q_{i}+\sum_{m=1}^{k_{0}+\ell} u_{m} \cdot 3^{-m}, v^{\ell}=r_{i}+\sum_{m=1}^{\ell} v_{m} \cdot 3^{-m} .
$$

(C3): $u_{k_{0}+1}, \ldots, u_{k_{0}+\ell} \in\{0,2\}$ and $v_{1}, \ldots, v_{\ell} \in\{0,2\}$.

In this way we will obtain

$$
R_{i}^{*}:=R_{i}\left(u^{2 k_{0}}, v^{k_{0}}\right) \in \mathcal{R}\left(2 k_{0}, k_{0}\right) .
$$

It is clear by the definition that the following two assertions hold:

Remark 28. (1) Using Fact 20, all the $3^{k_{0}}$ level $2 k_{0}$ grid squares contained in $R_{i}^{*}$ are in $\widetilde{F}^{2 k_{0}}$.

(2) The slope of the increasing diagonal of the rectangle $R_{i}^{*}$ is $3^{k_{0}}$, which is slightly larger than $M / N$. Thus every line of slope $M / N$ that enters the rectangle via the bottom horizontal line will leave $R_{i}^{*}$ through its Eastern side. The rectangle $R_{i}^{*}$ corresponds, in the simpler case $M / N<1, k_{0}=0$, to the whole square $\left(q_{i}, r_{i}\right)+I^{2}$ for which every line of slope $M / N$ that meets it must cross its Western or Eastern side.

Definition 29. Using the previous notation of this section, especially (51) and (50), we define the intervals

$$
J_{0}^{n}(i)=J_{R}\left(u^{2 k_{0}} 3^{2 k_{0}}\right), \quad J_{2}^{n}(i)=J_{L}\left(u^{2 k_{0}} 3^{2 k_{0}}+1\right) .
$$

The properties of these intervals, which are summarized in the following lemma, are immediate consequences of the definition. 
Lemma 30. (a): The orthogonal projection of $S_{a_{1} \ldots a_{n}} \cap R_{i}^{*}$ (the rectangle $R_{i}^{*}$ was defined in (52)) to the $x$-axis contains at least one of the level $n^{*}$ grid intervals $J_{0}^{n}(i)$ or $J_{2}^{n}(i)$. If the line Bottom $_{a_{1} \ldots a_{n}}$ enters the rectangle $R_{i}^{*}$ on its Western side, then $J_{0}^{n}(i)$ is such an interval. If the line Bottom $a_{a_{1} \ldots a_{n}}$ enters the rectangle $R_{i}^{*}$ on its Southern side, then $J_{2}^{n}(i)$ is such an interval.

(b): Using the notation of (51), by Fact 20, all the $3^{n^{*}-k_{0}}$ level $n^{*}$ grid squares both in $J_{0}^{n}(i) \times\left[v^{k_{0}}, v^{k_{0}}+3^{-k_{0}}\right]$ and in $J_{2}^{n}(i) \times\left[v^{k_{0}}, v^{k_{0}}+3^{-k_{0}}\right]$ are contained in $\widetilde{F}^{n^{*}}$.

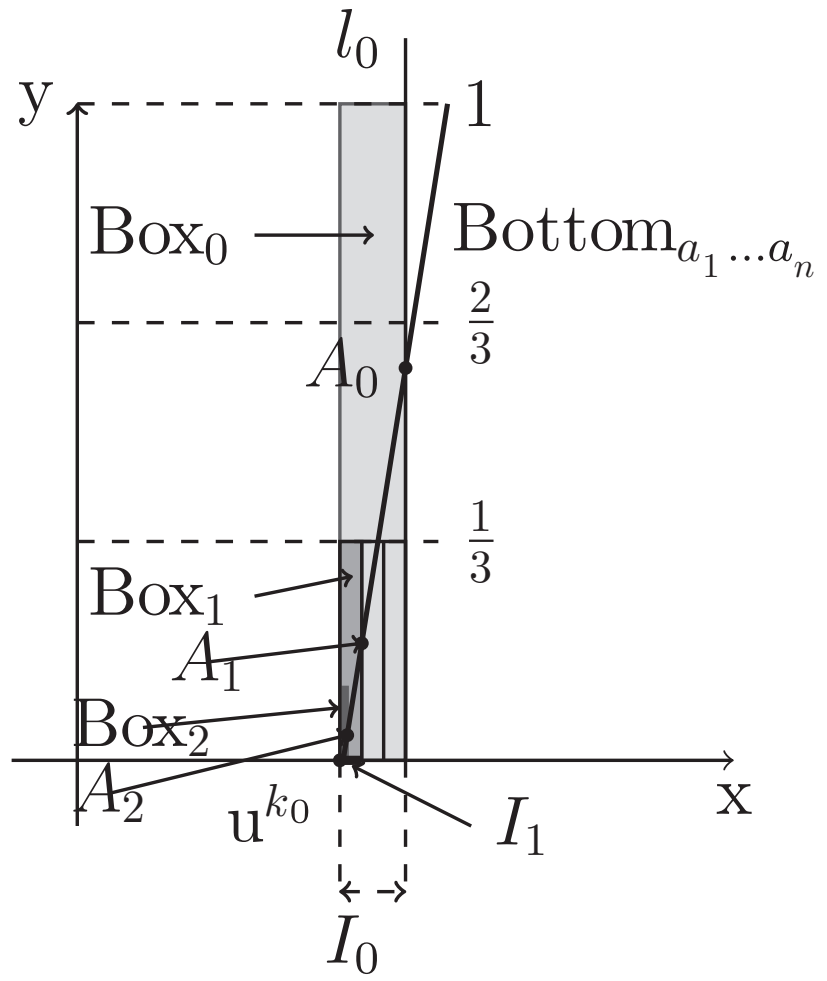

Figure 6. The inductive definition of $\operatorname{Box}_{\ell}$.

Now we present the inductive construction of rectangle $R_{i}^{*}$. Fix an $1 \leq i \leq K$ such that $Q_{i}$ is a relevant shape for $S_{a_{1} \ldots a_{n}}$. We recall that the line Bottom $a_{a_{1} \ldots a_{n}}$ was defined as the bottom edge of the strip $S_{a_{1} \ldots a_{n}}$. Using the notation of Definition 19 we construct a nested sequence of rectangles $\left\{R_{i}\left(u^{k_{0}+\ell}, v^{\ell}\right)\right\}_{\ell=0}^{k_{0}}$ satisfying the conditions (C1), (C2) and (C3). To construct $R_{i}\left(u^{k_{0}}, v^{0}\right)$ note that it follows from (43) that we can find a $0 \leq m^{\prime} \leq 3^{k_{0}}$ such that the line Bottom $a_{a_{1} \ldots a_{n}}$ intersects the vertical line segments $\left\{(x, y): x=q_{i}+m^{\prime} 3^{-k_{0}}, q_{i}<y<q_{i}+1\right\}$. Let us call this segment $l_{0}$ and the point where $l_{0}$ intersects Bottom $_{a_{1} \ldots a_{n}} A_{0}$. See Figure 6. Observe that $q_{i}+m^{\prime} 3^{-k_{0}}$ is the (left or right) end point of two level $k_{0}$ grid intervals. At least one of these two intervals lies in $\left[q_{i}, q_{i}+1\right]$. We call this level $k_{0}$ grid interval $I_{0}$ (if there are two such intervals, then we pick one). Note that the left end point of $I_{0}$ (which is either $q_{i}+m^{\prime} 3^{-k_{0}}$ or $q_{i}+m^{\prime} 3^{-k_{0}}-3^{-k_{0}}$ ) is defined as 
$u^{k_{0}}=q_{i}+\sum_{m=1}^{k_{0}} u_{m} 3^{-m}$. Let $v^{0}:=r_{i}$. Note that $A_{0}$ lies on one of the vertical sides of $\operatorname{Box}_{0}:=R_{i}\left(u^{k_{0}}, v^{0}\right)$. If the first ternary digit of the $y$-coordinate of $A_{0}$ is either 0 or 2 , then we call it $v_{1}$ and define $\operatorname{Box}_{1} \subset \operatorname{Box}_{0}$ as that $1 / 3$ scaled copy of $\operatorname{Box}_{0}$ which contains $A_{0}$ on one of its vertical sides. Clearly, the orthogonal projection $I_{1}$ of $\mathrm{Box}_{1}$ to the $x$-axis is a level $k_{0}+1$ grid interval which has a level $k_{0}$ end point. Therefore the last digit, called $u_{k_{0}+1}$, of the ternary representation of $I_{1}$ is either 0 or 2. In this way we defined $R_{i}\left(u^{k_{0}+1}, v^{1}\right)=\operatorname{Box}_{1}$. On the other hand, if the first ternary digit of the $y$-coordinate of $A_{0}$ is equal to 1 , then the definition of $u_{k_{0}+1}, v_{1}$ is more complicated. Namely, in this case we define Box ${ }_{1}$ as follows: Without loss of generality we may assume that $\mathrm{Box}_{0}$ is on the left hand side of $l_{0}$ (as in Figure 6). In this case we define $v_{1}:=0$ (otherwise we would have chosen $v_{1}=2$ ). We divide the bottom third part of $\mathrm{Box}_{0}$ into three equal vertical strips corresponding to the level $k_{0}+1$ grid intervals contained in $I_{0}$ (Figure 6). It follows from (43) that one of the vertical sides of one of these three $3^{-\left(k_{0}+1\right)} \times 3^{-1}$ rectangles, which is different from the middle one, intersects Bottom $_{a_{1} \ldots a_{n}}$. Let us call this point $A_{1}$, and the non-middle positioned $3^{-\left(k_{0}+1\right)} \times 3^{-1}$ grid rectangle which contains $A_{1}$ on one of its vertical sides is called $\mathrm{Box}_{1}$, and the projection of $\operatorname{Box}_{1}$ to the $x$-axis is called $I_{1}$. Clearly, $I_{1} \subset I_{0}$ is a level $3^{k_{0}+1}$ grid interval and its $k_{0}+1$ ternary digit is different from 1. This follows from the non-middle position of $\mathrm{Box}_{1}$ as mentioned above. So, the rectangle $R_{i}\left(u^{k_{0}+1}, v^{1}\right):=\operatorname{Box}_{1}$ satisfies the requirements (C1)-(C3). We continue the construction $R_{i}\left(u^{k_{0}+\ell}, v^{\ell}\right):=\operatorname{Box}_{\ell}$ for all $\ell \leq k_{0}$ exactly in the same way.

3.2.4. The proof of Proposition 18, Level $n$ shapes are labeled using $N \cdot \ell_{1, n}$ and which of these appear in $\tilde{F}^{n}$ is affected by $u_{1, n}$, so we compare these, using (34) and Fact 21, in the following lemma, which is the key step in proving Proposition 18

Lemma 31. Fix any $i, j \in\{1, \ldots, K\}$ satisfying $q_{i} \sim q_{j}$. Let $n \geq m_{0} \cdot N+1$. Assume that we are given

$$
u_{1}, \ldots, u_{n-\left(m_{0} N+1\right)} \in\{0,1,2\}
$$

in such a way that

$$
u_{1}+\cdots+u_{n-\left(m_{0} N+1\right)} \text { is an even number. }
$$

We can choose $u_{n-m_{0} N}, \ldots, u_{n} \in\{0,1,2\}$ and $\left(\ell_{1}, \ldots, \ell_{n}\right) \in\{0,1,2\}^{n}$ such that

$$
q_{j}+N \cdot \ell_{1, n}=3^{n} \cdot q_{i}+\sum_{k=1}^{n} 3^{n-k} \cdot u_{k} .
$$

Proof. Clearly we can choose $\left(\ell_{1}^{\prime}, \ldots, \ell_{n}^{\prime}\right) \in\{0,1,2\}^{n}$ such that for $p:=N\left(\ell_{1}^{\prime} \cdot 3^{n-1}+\cdots+\ell_{n}^{\prime}\right)-3^{n} \cdot q_{i}+q_{j}-\left(3^{n-1} \cdot u_{1}+\cdots+3^{m_{0} \cdot N+1} \cdot u_{n-\left(m_{0} \cdot N+1\right)}\right)$

we have

$$
0 \leq p \leq N-1 .
$$

Now we distinguish two cases based on the parity of $N$. 
$N$ is odd: Then it follows from (42) that

$$
\left\{2 \cdot\left(3^{m_{0}}+3^{2 m_{0}}+\cdots+3^{k m_{0}}\right)\right\}_{k=1}^{N}
$$

is a complete residue system modulo $N$ since $\{2 k\}_{k=1}^{N}$ is such a system. So we can find integers $1 \leq k \leq N$ and $v \in \mathbb{N}$ such that

$$
2\left(3^{m_{0}}+\cdots+3^{k m_{0}}\right)=v \cdot N+p .
$$

Choose $\left(\ell_{1}, \ldots, \ell_{n}\right) \in\{0,1,2\}^{n}$ such that

$$
N\left(\ell_{1}^{\prime} \cdot 3^{n-1}+\cdots+\ell_{n}^{\prime}\right)+v N=N\left(\ell_{1} \cdot 3^{n-1}+\cdots+\ell_{n}\right) .
$$

Then

$$
\begin{array}{r}
N\left(\ell_{1} \cdot 3^{n-1}+\cdots+\ell_{n}\right)-3^{n} \cdot q_{i}+q_{j}-\left(3^{n-1} \cdot u_{1}+\cdots+3^{m_{0} \cdot N+1} \cdot u_{n-\left(m_{0} \cdot N+1\right)}\right) \\
=v \cdot N+p=2\left(3^{m_{0}}+\cdots+3^{k m_{0}}\right),
\end{array}
$$

which immediately implies the assertion of the lemma.

$N$ is even: In this case

$$
\left\{2 \cdot\left(3^{m_{0}}+3^{2 m_{0}}+\cdots+3^{k m_{0}}\right)\right\}_{k=1}^{N}
$$

is not a complete residue system but contains (actually twice) all the even number residues. On the other hand, using $q_{i} \sim q_{j}$ and (54), we see that $p$ is an even number. This follows from (50) and Definition 29. So, as above, we can find integers $1 \leq k \leq N$ and $v \in \mathbb{N}$ such that (57) holds. The rest of the proof is the same as in the case when $N$ is odd.

This implies

Corollary 32. Fix an arbitrary $\left(a_{1}, \ldots, a_{n}\right) \in\{0,1,2\}^{n}$ and also fix $1 \leq i, j \leq K$ such that $q_{i} \sim q_{j}$. For any $u \in\{0,2\}$ we can find $\left(\ell_{1}, \ldots, \ell_{n}\right) \in\{0,1,2\}^{n}$ such that for the first component function $\left(\psi_{a_{1} \ldots a_{n}}^{\ell_{1} \ldots \ell_{n}}\right)_{1}$ of $\psi_{a_{1} \ldots a_{n}}^{\ell_{1} \ldots \ell_{n}}$ we have

(a): $\left(\psi_{a_{1} \ldots a_{n}}^{\ell_{1} \ldots \ell_{n}}\right)_{1}\left(q_{j}\right) \in J_{u}^{n}(i)$ and

(b): $Q_{a_{1} \ldots a_{n}}^{\ell_{1} \ldots \ell_{n}}(j) \subset \widetilde{F}^{n}$.

Proof. Using Fact 21 and Lemma 31 the proof immediately follows from the observation that for $u=0,2$, choosing $\left(\ell_{1}, \ldots, \ell_{n}\right)$ as in Lemma 31 we get that the first coordinate of $\psi_{a_{1} \ldots a_{n}}^{\ell_{1} \ldots \ell_{n}}\left(q_{j}, r_{j}\right)$ is

$$
\begin{aligned}
& \left(\psi_{a_{1} \ldots a_{n}}^{\ell_{1} \ldots \ell_{n}}\left(q_{j}, r_{j}\right)\right)_{1}=\frac{1}{3^{n}}\left(q_{j}+N\left(\ell_{1} \cdot 3^{n-1}+\cdots+\ell_{n}\right)\right) \\
& =q_{i}+u \cdot\left(3^{-1}+\cdots+3^{m_{0} \cdot N+1-n}\right)+2 \cdot 3^{-n}\left(3^{m_{0}}+\cdots+3^{k m_{0}}\right),
\end{aligned}
$$

where $0 \leq k \leq N-1$.

Lemma 33. Assume that $S_{a_{1} \ldots a_{n}}$ is an n-good strip. For each $1 \leq i, j \leq K$ let $\mathbf{r}_{i}$ be the $i$-th row vector of the matrix $A_{a_{1} \ldots a_{n}}$ and write $\mathbf{r}_{i}(j)$ for the $j$-th element of $\mathbf{r}_{i}$. Then

$$
\mathbf{r}_{i} \neq \mathbf{0} \text { and } q_{j} \sim q_{i} \text { imply } A_{a_{1} \ldots a_{n}}(i, j)=\mathbf{r}_{i}(j)>0 .
$$


Proof. Recall

$$
\mathbf{r}_{i}(j)=\#\left\{\left(\ell_{1}, \ldots, \ell_{n}\right) \in\{0,1,2\}^{n} \mid Q_{a_{1} \ldots a_{n}}^{\ell_{1} \ldots \ell_{n}}(j) \subset Q_{i} \cap \widetilde{F}^{n}\right\} .
$$

Assume that $\operatorname{int}\left(Q_{i} \cap S_{a_{1} \ldots a_{n}}\right) \neq \emptyset$. It is enough to prove that

$$
j \leq K, q_{i} \sim q_{j} \Rightarrow \exists\left(\ell_{1}, \ldots, \ell_{n}\right) \in\{0,1,2\}^{n} \text { s. t. } Q_{a_{1} \ldots a_{n}}^{\ell_{1} \ldots \ell_{n}}(j) \subset Q_{i} \cap \widetilde{F}^{n} .
$$

Namely, by definition,

$$
Q_{a_{1} \ldots a_{n}}^{\ell_{1} \ldots \ell_{n}}(j) \cap S_{a_{1} \ldots a_{n}}^{\ell_{1} \ldots \ell_{n}} \subset S_{a_{1} \ldots a_{n}} .
$$

To verify (61) we fix $j \leq K$ such that $q_{i} \sim q_{j}$. Let $u \in\{0,2\}$ be chosen such that assumption (A2) holds for $J_{u}^{n}(i)$. For this $u, i, j$ and $\left(a_{1}, \ldots, a_{n}\right)$ we choose an $\left(\ell_{1}, \ldots, \ell_{n}\right) \in\{0,1,2\}^{n}$ which satisfies Corollary 32. This implies that we have $Q_{a_{1} \ldots a_{n}}^{\ell_{1} \ldots \ell_{n}}(j) \subset Q_{i} \cap \widetilde{F}^{n}$. Namely, $\left(\psi_{a_{1} \ldots a_{n}}^{\ell_{1} \ldots \ell_{n}}\right)_{1}\left(q_{j}\right) \in J_{k}(i)$.

Corollary 34. We assume that $S_{a_{1} \ldots a_{n}}$ is an n-good strip and we write $\mathbf{r}_{i}$ for the $i$-th row vector of the matrix $A_{a_{1} \ldots a_{n}}$. Assume that $\mathbf{r}_{i} \neq \mathbf{0}$.

(a): If $N$ is odd, then all the elements of $\mathbf{r}_{i}$ are positive,

(b): If $N$ is even, then for all $j$ satisfying $q_{j} \sim q_{i}$, we have $\mathbf{r}_{i}(j)>0$.

Note that for a shape $Q_{i}$ which is relevant for the $n$-good strip $S_{a_{1} \ldots a_{n}}$ we have $\mathbf{r}_{i} \neq \mathbf{0}$.

3.3. The case when $N$ is an even number. The above argument shows that in the case when $3 \nmid N$ we can find $a_{1}, \ldots, a_{n}$ such that all the rows of some matrices $A_{a_{1} \ldots a_{n}}$ are either all-positive or all-zero. Now we would like to add to Corollary 34 (b) and prove the same in the case when $N$ is even.

In this section we always assume that $N$ is even. We fix $n$ which is large enough. (It will be specified later how large $n$ has to be.) We always assume that $\mathbf{a}=$ $\left(a_{1}, \ldots, a_{n}\right) \in\{0,1,2\}^{n}$ is chosen in such a way that $a_{n}=0$ and $S_{a_{1} \ldots a_{n}}$ is an $n$-good strip. For every $q \in\{0, \ldots, N-1\}$ we can find a unique $i=i(q, \mathbf{a}) \in\{1, \ldots, K\}$ such that:

(a): $q=q_{i}$.

(b): $Q_{i}$ is a relevant shape for $S_{a_{1} \ldots a_{n}}$.

(c): For $J(q):=\left[q+\frac{1}{3^{n}}, q+\frac{2}{3^{n}}\right]$ we have

$$
J(q) \subset J_{0}(i) \subset(q, q+1) \cap \pi_{1}\left(e_{1}\left(i, a_{1}, \ldots, a_{n}\right)\right) \cap \pi_{1}\left(e_{2}\left(i, a_{1}, \ldots, a_{n}\right)\right),
$$

where $\pi_{1}$ is the projection to the first axis.

Observe that

$$
J(q)=q+\left[\sum_{k=1}^{n} u_{k} 3^{-k}, \sum_{k=1}^{n} u_{k} 3^{-k}+3^{-n}\right], \text { where } u_{1}=\cdots=u_{n-1}=0, u_{n}=1 .
$$

One of the motivations to consider the intervals $J(q)$ is as follows.

Fact 35. For some $0 \leq q \leq N-1$ let $C=J(q) \times J^{\prime}$, where

$$
J^{\prime}:=m+\left[\sum_{k=1}^{n} v_{k} 3^{-k}, \sum_{k=1}^{n} v_{k} 3^{-k}+\frac{1}{3^{n}}\right],
$$


for some $0 \leq m \leq M-1$. Then

$$
C \subset(q, m)+F^{n} \text { if and only if } v_{n} \neq 1,
$$

where $F^{n}$ is the $n$-th approximation of $F$.

The proof of this fact is an immediate corollary of Fact 21] and (63).

Given $n$ and $q$ define $\ell_{1}, \ldots, \ell_{n} \in\{0,1,2\}^{n}$ as follows:

$$
\left\lfloor\frac{3^{n} q+1}{N}\right\rfloor=\ell_{1} \cdot 3^{n-1}+\cdots+\ell_{n-1} \cdot 3+\ell_{n} .
$$

Then we have

$$
J(q) \subset\left[N \cdot\left(\frac{\ell_{1}}{3}+\cdots+\frac{\ell_{n}}{3^{n}}\right), N \cdot\left(\frac{\ell_{1}}{3}+\cdots+\frac{\ell_{n}}{3^{n}}+\frac{1}{3^{n}}\right)\right] .
$$

Thus there is a unique $z_{1}=z_{1}(q) \in\{0, \ldots, N-1\}$ such that

$$
z_{1}(q):=3^{n} \cdot q+1-N \cdot 3^{n} \cdot\left(\frac{\ell_{1}}{3}+\cdots+\frac{\ell_{n}}{3^{n}}\right) .
$$

(Thus $z_{1}(q)$ is the number of the interval $J(q)$ when we count modulo $N$ the horizontal intervals of width $3^{-n}$.) It is easy to see that $q \rightarrow z_{1}(q)$ is a bijection on $\{0, \ldots, N-1\}$. Since $N$ is even we obtain

$$
\forall q \in\{0, \ldots, N-1\}, \quad q \nsim z_{1}(q) .
$$

Further, since $3 \nmid N$, the map $z_{1}:\{0, \ldots, N-1\} \rightarrow\{0, \ldots, N-1\}$ is a bijection. Now we define $z_{2}:=z_{2}(q) \in\{0, \ldots, M-1\}$ as follows:

(69) $z_{2}(q):=\min \left\{k \mid\left(\left(z_{1}(q), k\right)+I^{2}\right) \cap \operatorname{int}(S) \neq \emptyset, k+M \cdot \ell_{n}(q) \not \equiv 1 \bmod 3\right\}$.

We write

$$
C(q, \mathbf{a}):=\psi_{a_{1} \ldots a_{n}}^{\ell_{1}(q) \ldots \ell_{n}(q)}\left(\left(z_{1}(q), z_{2}(q)\right)+I^{2}\right) .
$$

Using Fact 21 and $a_{n}=0$, by (34) we obtain

$$
C(q, \mathbf{a}) \subset Q_{i(q, \mathbf{a})} \cap \widetilde{F}^{n} \text { and } C(q, \mathbf{a}) \cap S_{a_{1} \ldots a_{n}} \neq \emptyset .
$$

Let $Q(q):=\left(\left(z_{1}(q), z_{2}(q)\right)+I^{2}\right) \cap S$. Note that we could choose $Q(q)$ independent of a only because we always assume here that we restrict our attention to those $n$-good strips $S_{a_{1} \ldots a_{n}}$ for which $a_{n}=0$. Then

$$
\psi_{a_{1} \ldots a_{n}}^{\ell_{1} \ldots \ell_{n}}(Q(q))=C(q, \mathbf{a}) \cap S_{a_{1} \ldots a_{n}} .
$$

Summarizing what we have proved above:

Lemma 36. Assuming that $S_{a_{1} \ldots a_{n}}$ is an n-good strip and $a_{n}=0$, for every $m \in\{0, \ldots, N-1\}$ we can find $1 \leq i, j \leq K$ such that $q_{i} \nsim q_{j}, q_{j}=m$ and $A_{a_{1} \ldots a_{n}}(i, j)>0$.

Proof. With $q:=z_{1}^{-1}(m), i:=i(q, \mathbf{a})$ and $Q_{j}:=Q(q)$ the assertion of the lemma follows.

Lemma 37. We can find $n$ and $\mathbf{a}=\left(a_{1}, \ldots, a_{n}\right) \in\{0,1,2\}^{n}$ with $a_{n}=0$ and $q^{\prime} \nsim q^{\prime \prime} \in\{0, \ldots, N-1\}$ such that $S_{a_{1}, \ldots, a_{n}}$ is an n-good strip and both $Q\left(q^{\prime}\right)$ and $Q\left(q^{\prime \prime}\right)$ are relevant shapes for $S_{a_{1}, \ldots, a_{n}}$. 
Proof. First suppose that $M / N \geq 1$. Then $Q\left(z_{1}^{-1}(0)\right)$ is either $Q_{1}=I^{2} \cap S$ or $Q_{2}=\left((0,1)+I^{2}\right) \cap S$. Each is relevant for any $n$-good strip $S_{a_{1}, \ldots, a_{n}}$. By Fact 25 we can now choose any $n$-good strip $S_{a_{1}, \ldots, a_{n}}$ with $a_{n}=0$ for which $Q\left(z_{1}^{-1}(1)\right)$ is relevant and it is also relevant for $Q\left(z_{1}^{-1}(0)\right)$. As $0 \nsim 1$ we have $z_{1}^{-1}(0) \nsim z_{1}^{-1}(1)$ as required.

Now suppose that $M / N<1$. Then $Q\left(z_{1}^{-1}(0)\right)$ is either $Q_{1}$ or $Q_{2}$. Clearly, if $Q\left(z_{1}^{-1}(0)\right)=Q_{1}$, then whichever way we choose $Q\left(z_{1}^{-1}(1)\right) \subset([1,2] \times \mathbb{R}) \cap S$ we can find a narrow enough strip of slope $M / N$ through this shape and $Q_{1}$. This would immediately give us by Fact 25 that $q^{\prime}=z_{1}^{-1}(0), q^{\prime \prime}=z_{1}^{-1}(1)$ satisfy the requirements of the lemma. So, we may assume that $Q\left(z_{1}^{-1}(0)\right)=Q_{2}$. By symmetry, for the same reason we can assume that $Q\left(z_{1}^{-1}(N-1)\right)=Q_{K-1}=$ $\left((N-1, M-1)+I^{2}\right) \cap S$. If $M / N>1 / 2$, then $M / N<(M-1) /(N-1)$ and so we can find a narrow enough strip of slope $M / N$ which traverses both $Q_{2}$ and $Q_{K-1}$. So, by Fact 25] we can choose $q^{\prime}=z_{1}^{-1}(0), q^{\prime \prime}=z_{1}^{-1}(N-1)$.

Now assume that $0<M / N<1 / 2$. First suppose that

$$
\begin{aligned}
& Q\left(z_{1}^{-1}(1)\right)=Q_{3}=\left((1,0)+I^{2}\right) \cap S \text { and } \\
& \qquad Q\left(z_{1}^{-1}(N-2)\right)=Q_{K-2}=\left((N-2, M)+I^{2}\right) \cap S .
\end{aligned}
$$

Then by elementary geometry one can find a narrow strip which intersects the interior of both $Q_{3}$ and $Q_{K-2}$. So, by Fact 25, we can choose $q^{\prime}=z_{1}^{-1}(1)$ and $q^{\prime \prime}=z_{1}^{-1}(N-2)$.

If (73) does not hold, then either

$$
Q\left(z_{1}^{-1}(1)\right)=Q_{4}=\left((1,1)+I^{2}\right) \cap S
$$

Or

$$
Q\left(z_{1}^{-1}(N-2)\right)=Q_{K-3}=\left((N-2, M-1)+I^{2}\right) \cap S .
$$

If (74) holds we put $q^{\prime}=z_{1}^{-1}(0), q^{\prime \prime}=z_{1}^{-1}(1)$ and if (75) holds we put $q^{\prime}=$ $z_{1}^{-1}(N-2), q^{\prime \prime}=z_{1}^{-1}(N-1)$.

Now we are ready to prove Proposition 18

Proof of Proposition 18. If $N$ is odd, then the assertion of the proposition follows from Lemma 33. So, we may assume that $N$ is even. Let us fix $n$ and $\widetilde{\mathbf{a}}=$ $\left(\widetilde{a}_{1}, \ldots, \widetilde{a}_{n}\right)$, and $q^{\prime}, q^{\prime \prime}$ whose existence is guaranteed by Lemma 37 . Using the notation of Lemma [36 we substitute $m=q^{\prime}$ to define $i^{\prime}, j^{\prime}$ as in Lemma 36. Further, we also substitute $m=q^{\prime \prime}$ in Lemma 36 to define $i^{\prime \prime}, j^{\prime \prime}$. As a shorthand notation we write in this proof

$$
A:=A_{\widetilde{a}_{1}, \ldots, \widetilde{a}_{n}} .
$$

Then by definition we have

$$
A\left(i^{\prime}, j^{\prime}\right), A\left(i^{\prime \prime}, j^{\prime \prime}\right)>0
$$

Without loss of generality we may assume that

$$
q^{\prime}=q_{j^{\prime}} \text { and } q_{i^{\prime \prime}} \text { are even and } q^{\prime \prime}=q_{j^{\prime \prime}} \text { and } q_{i^{\prime}} \text { are odd. }
$$

We will use the following observation: it follows from Corollary 34(b) that for any $1 \leq k, \ell \leq K$ we have

$$
A^{2}(k, \ell) \geq A(k, k) \cdot A(k, \ell) \geq A(k, \ell),
$$


since all the non-zero entries of $A$ are at least one. First we prove that

$$
A^{2}\left(i^{\prime}, k\right)>0 \text { and } A^{2}\left(i^{\prime \prime}, k\right)>0 \text { for every } 1 \leq k \leq K .
$$

To see that the first inequality holds observe that it follows from (76) and Corollary 34 (b) that whenever $q_{k}$ is even we have

$$
A^{2}\left(i^{\prime}, k\right) \geq A\left(i^{\prime}, j^{\prime}\right) \cdot A\left(j^{\prime}, k\right)>0 .
$$

Similarly if $q_{k}$ is an odd number, then using (78) and Corollary 34 (b) we get

$$
A^{2}\left(i^{\prime}, k\right) \geq A\left(i^{\prime}, k\right)>0,
$$

which completes the proof of the first half of (78). By symmetry, the second inequality in (78) can be proved in the same way.

Now let $u \in\{1, \ldots, K\}$ be arbitrary such that the $u$-th row of $A$ is a non-all-zero row. To prove Proposition 18 it is enough to show that for every $v \in\{1, \ldots, K\}$ we have

$$
A^{3}(u, v)>0 .
$$

If $q_{u}, q_{v}$ have the same parity, then this follows from Corollary 34 (b) and (77). If $q_{u}$ and $q_{v}$ have different parity, then without loss of generality we assume that $q_{u}$ is odd. Since we assumed that $q_{i^{\prime}}$ is also odd we get from Corollary 34 (b) that $A\left(u, i^{\prime}\right)>0$. So, (78) yields that

$$
A^{3}(u, v) \geq A\left(u, i^{\prime}\right) \cdot A^{2}\left(i^{\prime}, v\right)>0 .
$$

Thus we have verified Proposition 18 with $n_{0}:=3 n$ and

$$
\left(a_{1}, \ldots, a_{n_{0}}\right)=\left(\widetilde{a}_{1}, \ldots, \widetilde{a}_{n}, \widetilde{a}_{1}, \ldots, \widetilde{a}_{n}, \widetilde{a}_{1}, \ldots, \widetilde{a}_{n}\right) .
$$

3.4. A corollary of the Perron-Frobenius theorem. We fix $n_{0}$ and $A_{a_{1} \ldots a_{n_{0}}}$ which satisfies Proposition 18. We consider the matrices

$$
\mathcal{B}:=\left\{A_{i_{1} \ldots i_{n_{0}}}\right\}_{\left(i_{1} \ldots i_{n_{0}}\right) \in\{0,1,2\}^{n_{0}}} .
$$

We write $T:=3^{n_{0}}$ and $\mathcal{B}=\left\{B_{1}, \ldots, B_{T}\right\}$, where

$$
B_{1}:=A_{a_{1} \ldots a_{n_{0}}} .
$$

Put

$$
B_{s}:=\sum_{k=1}^{T} B_{k}
$$

Since

$$
B_{s}=\sum_{i_{1} \ldots i_{n_{0}}} A_{i_{1} \ldots i_{n_{0}}}=\left(A_{0}+A_{1}+A_{2}\right)^{n_{0}},
$$

using (19) one immediately gets that

$$
B_{s} \text { has each column sum } 8^{n_{0}} .
$$

Further,

$$
\forall 1 \leq k \leq T \text {, each column sum of } B_{k} \in\left[2^{n_{0}}, 3^{n_{0}}\right] .
$$

In particular all the matrices $B_{k}$ are column allowable (every column contains a non-zero element) non-negative integer matrices. 
We define the Lyapunov exponent for the random product of the matrices $\left\{B_{i}\right\}_{i=1}^{T}$, where for each $i$ in every step we choose $B_{i}$ independently with probability $1 / T$. Using (21) the Lyapunov exponent

$$
\gamma_{B}:=\lim _{n \rightarrow \infty} \frac{1}{n} \log \left\|B_{i_{1} \ldots i_{n}}\right\|_{1}, \text { for a.a. }\left(i_{1}, i_{2}, \ldots\right) .
$$

Then clearly we have

$$
\gamma=\frac{1}{n_{0}} \cdot \gamma_{B}
$$

where we recall that $T=3^{n_{0}}$. Note that it follows from (22) that

$$
\lim _{k \rightarrow \infty} \frac{1}{k} \cdot \sum_{j_{1} \ldots j_{k}} \frac{1}{T^{k}} \cdot \log \left\|B_{j_{1} \ldots j_{k}}\right\|_{1}=\gamma_{B}
$$

Let $\widehat{B}_{1}$ be the matrix that we obtain from $B_{1}$ when we replace all the column vectors of $B_{1}$ that correspond to an all-zero row by all-zero columns. That is, if the column and row vectors of $B_{1}$ are

$$
B_{1}=\left[\mathbf{c}_{1}, \ldots, \mathbf{c}_{K}\right] \text { and } B_{1}=\left[\mathbf{r}_{1}, \ldots, \mathbf{r}_{K}\right] \text {, }
$$

then the matrix $\widehat{B}_{1}$ is defined by its column vectors as follows:

$$
\widehat{B}_{1}=\left[\mathbf{c}_{1}^{*}, \ldots, \mathbf{c}_{K}^{*}\right]
$$

where

Note that for any $k$ we have

$$
\mathbf{c}_{i}^{*}= \begin{cases}\mathbf{c}_{i}, & \text { if } \mathbf{r}_{i}>\mathbf{0} \\ \mathbf{0}, & \text { if } \mathbf{r}_{i}=\mathbf{0}\end{cases}
$$

$$
B_{1}^{k+1}=\widehat{B}_{1}^{k} \cdot B_{1}
$$

Let

$$
\ell:=\#\left\{i: \mathbf{c}_{i}>\mathbf{0}\right\}
$$

We choose an orthogonal matrix $Q$ that corresponds to a change of the order of the basis vectors in the natural basis such that

$$
Q \cdot \widehat{B}_{1} \cdot Q^{T}=\left[\begin{array}{l|l}
C & \mathbf{0} \\
\hline \mathbf{0} & \mathbf{0}
\end{array}\right],
$$

where $C$ is a positive (all the elements are positive) $\ell \times \ell$ matrix. It follows from the Perron-Frobenius theorem [1, p. 185], 17, p. 9] that for the leading eigenvalue $\rho$ and normalized left and right leading eigenvectors $\mathbf{u}, \mathbf{v} \in \mathbb{R}^{\ell}$ of $C$ we have

$$
\mathbf{u}^{T} \cdot C=\rho \cdot \mathbf{u}^{T}, C \cdot \mathbf{v}=\rho \cdot \mathbf{v}, \mathbf{u}>\mathbf{0}, \mathbf{v}>\mathbf{0}, \sum_{i=1}^{\ell} u_{i} \cdot v_{i}=1, \sum_{i=1}^{\ell} v_{i}=1 .
$$

Furthermore, there exists $0<\rho_{0}<\rho$ and $\ell \times \ell$ matrices $R^{k}$ such that for the $\ell \times \ell$ matrix

$$
P:=\mathbf{v} \cdot \mathbf{u}^{T}
$$

we have

$$
\forall k \quad C^{k}=\rho^{k} \cdot P+R^{k},
$$

where for all $1 \leq i, j \leq K$ the $(i, j)$-th element $r_{i, j}^{(k)}$ of the matrix $R^{k}$ satisfies

$$
\left|r_{i, j}^{(k)}\right|<c_{1} \cdot \rho_{0}^{k},
$$


for some constant $c_{1}>0$. Sometimes we will have to extend vectors defined in $\mathbb{R}^{\ell}$ to $\mathbb{R}^{K}$. We will do this in two different ways: namely, for $\mathbf{a} \in \mathbb{R}^{\ell}$ we write

$$
\mathbf{a}_{*}:=\left[\begin{array}{l}
\mathbf{a} \\
\hline \mathbf{0}
\end{array}\right] \text { and } \mathbf{a}^{*}:=Q^{-1} \mathbf{a}_{*} .
$$

The meaning of $\mathbf{a}^{*}$ is as follows: to provide that all the $\ell$ positive rows of $B_{1}$ become the first $\ell$ rows we needed to use a permutation of the basis vectors of the natural basis. This permutation was provided by multiplying by the orthogonal matrix $Q$ on the left. To permute all the coordinates to the original order we have to multiply by $Q^{-1}$ on the left.

Remark 38. (a): In the following definition we use the fact that by the definition of $Q$ the last $K-\ell$ rows of the matrix $Q \cdot B_{1} \cdot Q^{T}$ are all-zero rows and the first $\ell$ rows are all-positive rows. That is, there exists an $\ell \times K$ matrix $D$ such that

$$
Q \cdot B_{1} \cdot Q^{T}=\left[\frac{D}{\mathbf{0}}\right] \text { and } D=[C \mid E] \text { with } E>\mathbf{0} .
$$

(b): By the definition of $Q$ we have

$$
B_{1} Q^{-1} \mathbf{v}_{*}=Q^{-1}\left((C \cdot \mathbf{v})_{*}\right)=Q^{-1} \rho \mathbf{v}_{*}=Q^{-1}\left[\frac{C \mid E}{\mathbf{0}}\right] \mathbf{v}_{*}=\rho Q^{-1} \mathbf{v}_{*} .
$$

Note that $Q$ is an orthogonal matrix that corresponds to some change of the order in the natural basis. Hence $Q \geq \mathbf{0}$. In this way we see that $\rho$ is an eigenvalue of the matrix $B_{1}$. Furthermore, $\mathbf{v}^{*}=Q^{-1} \mathbf{v}_{*}$ is a non-negative eigenvector of the eigenvalue $\rho$.

Definition 39. For every $n$ and for every $\left(b_{1}, \ldots, b_{n}\right) \in\left\{1, \ldots, 3^{n_{0}}\right\}^{n}$ :

(a): We write $E_{b_{1} \ldots b_{n}}$ for the $\ell \times K$ matrix which satisfies

$$
\left[\frac{E_{b_{1} \ldots b_{n}}}{\mathbf{0}}\right]=Q \cdot B_{1} \cdot Q^{T} \cdot Q \cdot B_{b_{1} \ldots b_{n}} \cdot Q^{T}=Q \cdot B_{1} \cdot B_{b_{1} \ldots b_{n}} \cdot Q^{T} .
$$

(b): We define the positive vector of $K$ components

$$
\mathbf{u}_{b_{1} \ldots b_{n}}^{T}:=\mathbf{u}^{T} \cdot E_{b_{1} \ldots b_{n}} .
$$

(c): For $\varepsilon \in \mathbb{R}$ we define the (column) vector $\varepsilon \in \mathbb{R}^{\ell}$ as a vector with all components $\varepsilon$.

Lemma 40. Let $0<\varepsilon_{1}<\min _{1 \leq i<\ell} v_{i}$. Then there exists $k_{0}$ such that for every $n$ and for every $\left(b_{1}, \ldots, b_{n}\right) \in\left\{1, \ldots, 3^{n_{0}}\right\}^{n}$ we have

$$
\rho^{k_{0}} \cdot\left(\mathbf{v}-\frac{1}{10} \varepsilon_{1}\right) \cdot \mathbf{u}_{b_{1} \ldots b_{n}}^{T}<C^{k_{0}} \cdot E_{b_{1} \ldots b_{n}}<\rho^{k_{0}} \cdot\left(\mathbf{v}+\frac{1}{10} \varepsilon_{1}\right) \cdot \mathbf{u}_{b_{1} \ldots b_{n}}^{T} .
$$

Proof. The proof follows from (92). Namely,

$$
k_{0}:=k_{0}\left(\varepsilon_{1}\right) \text { is defined as } k_{0}:=\min \left\{k: c_{1} \cdot\left\{\frac{\rho_{0}}{\rho}\right\}^{k}<\frac{\varepsilon_{1}}{10} \cdot \min _{1 \leq i \leq \ell} u_{i}\right\} \text {. }
$$

Then by (92) we have $\frac{1}{\rho^{k_{0}}} \cdot\left|r_{i, j}^{\left(k_{0}\right)}\right|<\frac{\varepsilon_{1}}{10} \cdot \min u_{i}$. Hence

$$
\rho^{k_{0}}\left(\mathbf{v} \cdot \mathbf{u}^{T}-\frac{1}{10} \varepsilon_{\mathbf{1}} \cdot \mathbf{u}^{T}\right)<C^{k_{0}}<\rho^{k_{0}}\left(\mathbf{v} \cdot \mathbf{u}^{T}+\frac{1}{10} \boldsymbol{\varepsilon}_{\mathbf{1}} \cdot \mathbf{u}^{T}\right) .
$$


This completes the proof of the lemma because all the elements of all the matrices and vectors in the last inequality as well as in the assertion of the lemma are non-negative.

Lemma 41. There is $\left(i_{1}, \ldots, i_{n_{0}}\right) \in\{0,1,2\}^{n_{0}}$ such that

$$
\left\|A_{a_{1} \ldots a_{n_{0}}} \cdot \mathbf{v}^{*}\right\|_{1} \neq\left\|A_{i_{1} \ldots i_{n_{0}}} \cdot \mathbf{v}^{*}\right\|_{1},
$$

where we recall that $\mathbf{v}^{*}$ was defined by the convention introduced in (93).

Proof. To get a contradiction we assume that

$$
\forall\left(i_{1}, \ldots, i_{n_{0}}\right) \in\{0,1,2\}^{n_{0}},\left\|A_{a_{1} \ldots a_{n_{0}}} \cdot \mathbf{v}^{*}\right\|_{1}=\left\|A_{i_{1} \ldots i_{n_{0}}} \cdot \mathbf{v}^{*}\right\|_{1} .
$$

Then

$$
\begin{aligned}
3^{n_{0}} \cdot \rho & =3^{n_{0}} \cdot\left\|\rho \cdot \mathbf{v}^{*}\right\|_{1}=3^{n_{0}} \cdot\left\|B_{1} \cdot \mathbf{v}^{*}\right\|_{1} \\
& =\sum_{\left(i_{1}, \ldots, i_{n_{0}}\right)}\left\|A_{i_{1} \ldots i_{n_{0}}} \cdot \mathbf{v}^{*}\right\|_{1}=\left\|\sum_{\left(i_{1}, \ldots, i_{n_{0}}\right)} A_{i_{1} \ldots i_{n_{0}}} \cdot \mathbf{v}^{*}\right\|_{1} \\
& =\left\|A_{s}^{n_{0}} \cdot \mathbf{v}^{*}\right\|_{1}=8^{n_{0}}\left\|\mathbf{v}^{*}\right\|_{1}=8^{n_{0}},
\end{aligned}
$$

so $\rho=8^{n_{0}} / 3^{n_{0}}$. However this is impossible since $8^{n_{0}} / 3^{n_{0}}$ cannot be a root of the characteristic polynomial of $B_{1}$ which is a matrix of integer coefficients.

We assume from now on that we numbered the elements of $\mathcal{B}$ such that

$$
\left\|B_{1} \cdot \mathbf{v}\right\| \neq\left\|B_{2} \cdot \mathbf{v}\right\| .
$$

Without loss of generality we may assume that $\left\|B_{1} \cdot \mathbf{v}\right\|<\left\|B_{2} \cdot \mathbf{v}\right\|$.

Definition 42. Let us define $0<\varepsilon_{1}<\frac{1}{20} \cdot \min v_{i}$ and $c_{0}>0$ such that

$$
\frac{\left\|B_{1} \cdot\left(\mathbf{v}+10 \varepsilon_{\mathbf{1}}\right)^{*}\right\|_{1}}{\left\|\left(\mathbf{v}-10 \varepsilon_{\mathbf{1}}\right)\right\|_{1}}+c_{0}<\frac{\left\|B_{2} \cdot\left(\mathbf{v}-10 \varepsilon_{\mathbf{1}}\right)^{*}\right\|_{1}}{\left\|\left(\mathbf{v}+10 \varepsilon_{\mathbf{1}}\right)\right\|_{1}} .
$$

Lemma 43. Here we use the notation of Lemma 40, For an arbitrary $\left(b_{1}, \ldots, b_{n}\right) \in$ $\{0,1,2\}^{n}$ we define

$$
F_{b_{1} \ldots b_{n}}:=B_{1}^{k_{0}+1} \cdot B_{b_{1} \ldots b_{n}} .
$$

Then for an arbitrary $n$ and for arbitrary $\left(b_{1}, \ldots, b_{n}\right) \in\{0,1,2\}^{n}$ we have

$$
\frac{\left\|B_{1} \cdot F_{b_{1} \ldots b_{n}}\right\|_{1}}{\left\|F_{b_{1} \ldots b_{n}}\right\|_{1}}+c_{0}<\frac{\left\|B_{2} \cdot F_{b_{1} \ldots b_{n}}\right\|_{1}}{\left\|F_{b_{1} \ldots b_{n}}\right\|_{1}}
$$

where $\|A\|_{1}$ means the sum of the modulus of the elements of the matrix.

Proof. First we start with a simple observation that we will use at the end of this argument. Let $\mathbf{a}, \mathbf{b} \geq 0$ be vectors of $K$ components.

$$
\text { If } A=\mathbf{a} \cdot \mathbf{b}^{T} \text {, then }\|A\|_{1}=\|\mathbf{a}\|_{1} \cdot\left\|\mathbf{b}^{T}\right\|_{1} .
$$

Because $Q A Q^{T}$ is obtained from $A$ by permuting the rows and columns,

$$
\|A\|_{1}=\left\|Q \cdot A \cdot Q^{T}\right\|_{1} .
$$

Thus in order to verify (102) it is enough to estimate the ratio of the norms

$$
\left\|Q \cdot B_{j} \cdot F_{b_{1} \ldots b_{n}} \cdot Q^{T}\right\|_{1} \text { for } j=1,2 \text { and }\left\|Q \cdot F_{b_{1} \ldots b_{n}} \cdot Q^{T}\right\|_{1} .
$$


Using (89), (87) and Definition 39 (a) we obtain

$$
Q \cdot F_{b_{1} \ldots b_{n}} \cdot Q^{T}=\left[\begin{array}{c|c}
C^{k_{0}} & \mathbf{0} \\
\hline \mathbf{0} & \mathbf{0}
\end{array}\right] \cdot\left[\begin{array}{c}
E_{b_{1} \ldots b_{n}} \\
\hline \mathbf{0}
\end{array}\right]=\left[\begin{array}{c}
C^{k_{0}} \cdot E_{b_{1} \ldots b_{n}} \\
\hline \mathbf{0}
\end{array}\right] .
$$

Lemma 40 asserts that

$$
\rho^{k_{0}} \cdot\left(\mathbf{v}-\frac{1}{10} \varepsilon_{\mathbf{1}}\right) \cdot \mathbf{u}_{b_{1} \ldots b_{n}}^{T}<C^{k_{0}} \cdot E_{b_{1} \ldots b_{n}}<\rho^{k_{0}} \cdot\left(\mathbf{v}+\frac{1}{10} \varepsilon_{1}\right) \cdot \mathbf{u}_{b_{1} \ldots b_{n}}^{T},
$$

where we recall that $\mathbf{u}_{b_{1} \ldots b_{n}}^{T}=\mathbf{u}^{T} \cdot E_{b_{1} \ldots b_{n}}$. Using (103) this implies that (105)

$\rho^{k_{0}} \cdot\left\|\left(\mathbf{v}-\frac{1}{10} \varepsilon_{\mathbf{1}}\right)\right\|_{1} \cdot\left\|\mathbf{u}_{b_{1} \ldots b_{n}}^{T}\right\|_{1} \leq\left\|F_{b_{1} \ldots b_{n}}\right\|_{1} \leq \rho^{k_{0}} \cdot\left\|\left(\mathbf{v}+\frac{1}{10} \varepsilon_{\mathbf{1}}\right)\right\|_{1} \cdot\left\|\mathbf{u}_{b_{1} \ldots b_{n}}^{T}\right\|_{1}$.

Now we estimate

$$
\left\|Q \cdot B_{j} \cdot F_{b_{1} \ldots b_{n}} \cdot Q^{T}\right\|_{1}=\left\|Q \cdot B_{j} \cdot Q^{T} \cdot Q \cdot F_{b_{1} \ldots b_{n}} \cdot Q^{T}\right\|_{1}, \quad j=1,2 .
$$

Also from the assertion of Lemma 40 we obtain that

$$
\rho^{k_{0}} \cdot\left(\mathbf{v}-\frac{1}{10} \varepsilon_{1}\right)^{*} \cdot \mathbf{u}_{b_{1} \ldots b_{n}}^{T} \leq Q^{T} \cdot Q \cdot F_{b_{1} \ldots b_{n}} \cdot Q^{T} \leq \rho^{k_{0}} \cdot\left(\mathbf{v}+\frac{1}{10} \varepsilon_{\mathbf{1}}\right)^{*} \cdot \mathbf{u}_{b_{1} \ldots b_{n}}^{T} \cdot
$$

Using (103) again we get on the one hand,

$$
\left\|Q \cdot B_{j} \cdot F_{b_{1} \ldots b_{n}} \cdot Q^{T}\right\|_{1} \leq \rho^{k_{0}} \cdot\left\|B_{j} \cdot\left(\mathbf{v}+\frac{1}{10} \varepsilon_{1}\right)^{*}\right\|_{1} \cdot\left\|\mathbf{u}_{b_{1} \ldots b_{n}}^{T}\right\|_{1}
$$

and on the other hand,

$$
\left\|Q \cdot B_{j} \cdot F_{b_{1} \ldots b_{n}} \cdot Q^{T}\right\|_{1} \geq \rho^{k_{0}} \cdot\left\|B_{j} \cdot\left(\mathbf{v}-\frac{1}{10} \varepsilon_{1}\right)^{*}\right\|_{1} \cdot\left\|\mathbf{u}_{b_{1} \ldots b_{n}}^{T}\right\|_{1} .
$$

Putting together these last two inequalities with (105), the assertion of the lemma immediately follows from (101). Namely, for $j=1,2$,

$$
\frac{\left\|B_{j} \cdot\left(\mathbf{v}-\frac{1}{10} \varepsilon_{1}\right)^{*}\right\|_{1}}{\left\|\left(\mathbf{v}+\frac{1}{10} \varepsilon_{1}\right)\right\|_{1}} \leq \frac{\left\|B_{j} \cdot F_{b_{1} \ldots b_{n}}\right\|_{1}}{\left\|F_{b_{1} \ldots b_{n}}\right\|_{1}} \leq \frac{\left\|B_{j} \cdot\left(\mathbf{v}+\frac{1}{10} \varepsilon_{1}\right)^{*}\right\|_{1}}{\left\|\left(\mathbf{v}-\frac{1}{10} \varepsilon_{\mathbf{1}}\right)\right\|_{1}} .
$$

Lemma 44. For every $m$ and $b_{1}, \ldots, b_{m}$ and $i=1, \ldots, T$ we have

$$
\frac{\left\|B_{i} \cdot B_{b_{1} \ldots b_{m}}\right\|_{1}}{\left\|B_{b_{1} \ldots b_{m}}\right\|_{1}} \in\left[2^{n_{0}}, 3^{n_{0}}\right] .
$$

Proof. Using the notation and the assertion of Fact 16 and the fact that, for all $i$, every column sum of $B_{i}$ is between $2^{n_{0}}$ and $3^{n_{0}}$ we obtain

$$
\sum_{i=1}^{K} 2^{n_{0}}\left\|\mathbf{r}_{B_{b_{1} \ldots b_{n}}}(i)\right\|_{1} \leq\left\|B_{i} \cdot B_{b_{1} \ldots b_{m}}\right\|_{1} \leq \sum_{i=1}^{K} 3^{n_{0}}\left\|\mathbf{r}_{B_{b_{1} \ldots b_{n}}}(i)\right\|_{1} .
$$

The simple observation that $\left\|B_{b_{1} \ldots b_{n}}\right\|_{1}=\sum_{i=1}^{K}\left\|\mathbf{r}_{B_{b_{1} \ldots b_{n}}}(i)\right\|_{1}$ completes the proof of the lemma.

Definition $45 . \quad$ (a): Let

$$
R:=\left\{\left(x_{1}, \ldots, x_{T}\right) \in\left[2^{n_{0}}, 3^{n_{0}}\right]^{T}: \exists i, j \text { such that }\left|x_{i}-x_{j}\right| \geq c_{0}\right\},
$$


where $c_{0}>0$ is the constant defined in Lemma 43 . Using the well-known inequality between the arithmetic and geometric means we obtain that the continuous function

$$
f\left(x_{1}, \ldots, x_{T}\right):=\log \frac{\sum_{j=1}^{T} x_{j}}{T}-\log \sqrt[T]{x_{1} \cdots x_{T}}
$$

takes only positive values on the compact set $R$. We define

$$
\delta_{0}:=\left.\min f\right|_{R}>0
$$

(b): Let $m>k_{0}+1$. Put

$$
\mathcal{I}_{m}:=\{1, \ldots, T\}^{m}, \mathcal{I}_{m}^{\prime}:=\left\{\mathbf{i} \in \mathcal{I}_{m}: i_{1}=\cdots=i_{k_{0}+1}=1\right\} \text { and } \mathcal{I}_{m}^{\prime \prime}:=\mathcal{I}_{m} \backslash \mathcal{I}_{m}^{\prime} .
$$

Fact 46. For every $\mathbf{i} \in \mathcal{I}_{m}^{\prime}$ we have

$$
\frac{1}{T} \cdot \log \prod_{j=1}^{T} \frac{\left\|B_{j} \cdot B_{\mathbf{i}}\right\|_{1}}{\left\|B_{\mathbf{i}}\right\|_{1}}+\delta_{0} \leq \log \frac{1}{T} \sum_{j=1}^{T} \frac{\left\|B_{j} \cdot B_{\mathbf{i}}\right\|_{1}}{\left\|B_{\mathbf{i}}\right\|_{1}} .
$$

Proof. It follows from Lemma 43 that for every $\mathbf{i} \in \mathcal{I}_{m}^{\prime}$,

$$
\frac{\left\|B_{1} \cdot B_{\mathbf{i}}\right\|_{1}}{\left\|B_{\mathbf{i}}\right\|_{1}}+c_{0}<\frac{\left\|B_{2} \cdot B_{\mathbf{i}}\right\|_{1}}{\left\|B_{\mathbf{i}}\right\|_{1}}
$$

This and Lemma 44 imply that for every $\mathbf{i} \in \mathcal{I}_{m}^{\prime}$,

$$
\left(\frac{\left\|B_{1} \cdot B_{\mathbf{i}}\right\|_{1}}{\left\|B_{\mathbf{i}}\right\|_{1}}, \ldots, \frac{\left\|B_{T} \cdot B_{\mathbf{i}}\right\|_{1}}{\left\|B_{\mathbf{i}}\right\|_{1}}\right) \in R
$$

The assertion of the fact immediately follows from (111).

Observe that for every $\mathbf{i} \in \mathcal{I}_{m}$ we have

$$
\begin{aligned}
\sum_{j=1}^{T} \frac{\left\|B_{j} \cdot B_{\mathbf{i}}\right\|_{1}}{\left\|B_{\mathbf{i}}\right\|_{1}} & =\frac{\left\|\sum_{j=1}^{T} B_{j} \cdot B_{\mathbf{i}}\right\|_{1}}{\left\|B_{\mathbf{i}}\right\|_{1}} \\
& =\frac{\left\|B_{s} \cdot B_{\mathbf{i}}\right\|_{1}}{\left\|B_{\mathbf{i}}\right\|_{1}}=\frac{8^{n_{0}}\left\|B_{\mathbf{i}}\right\|_{1}}{\left\|B_{\mathbf{i}}\right\|_{1}}=8^{n_{0}},
\end{aligned}
$$

where in the next to last step we used (26) and (81). This and Fact 46 imply that

$$
\forall \mathbf{i} \in \mathcal{I}_{m}^{\prime}: \frac{1}{T} \cdot \log \prod_{j=1}^{T} \frac{\left\|B_{j} \cdot B_{\mathbf{i}}\right\|_{1}}{\left\|B_{\mathbf{i}}\right\|_{1}} \leq n_{0} \cdot \log \frac{8}{3}-\delta_{0} .
$$

Lemma 47. For every $m \geq k_{0}+1$ we have

$$
\frac{1}{T^{m}} \cdot \sum_{\mathbf{i} \in \mathcal{I}_{m}} \frac{1}{T} \log \prod_{j=1}^{T} \frac{\left\|B_{j} \cdot B_{\mathbf{i}}\right\|_{1}}{\left\|B_{\mathbf{i}}\right\|_{1}} \leq n_{0} \cdot \log \frac{8}{3}-T^{-\left(k_{0}+1\right)} \cdot \delta_{0} .
$$


Proof.

$$
\begin{aligned}
\frac{1}{T^{m}} \cdot \sum_{\mathbf{i} \in \mathcal{I}_{m}} \frac{1}{T} \log \prod_{j=1}^{T} \frac{\left\|B_{j} \cdot B_{\mathbf{i}}\right\|_{1}}{\left\|B_{\mathbf{i}}\right\|_{1}}= & \frac{1}{T^{m}} \cdot \sum_{\mathbf{i} \in \mathcal{I}_{m}} \frac{1}{T} \cdot \sum_{j=1}^{T} \log \frac{\left\|B_{j} \cdot B_{\mathbf{i}}\right\|_{1}}{\left\|B_{\mathbf{i}}\right\|_{1}} \\
= & \frac{1}{T^{m}} \cdot \sum_{\mathbf{i} \in \mathcal{I}_{m}^{\prime}} \frac{1}{T} \cdot \sum_{j=1}^{T} \log \frac{\left\|B_{j} \cdot B_{\mathbf{i}}\right\|_{1}}{\left\|B_{\mathbf{i}}\right\|_{1}} \\
& +\frac{1}{T^{m}} \cdot \sum_{\mathbf{i} \in \mathcal{I}_{m}^{\prime \prime}} \frac{1}{T} \cdot \sum_{j=1}^{T} \log \frac{\left\|B_{j} \cdot B_{\mathbf{i}}\right\|_{1}}{\left\|B_{\mathbf{i}}\right\|_{1}} \\
\leq & \frac{\# \mathcal{I}_{m}^{\prime}}{T^{m}} \cdot n_{0} \cdot \log \frac{8}{3}-\frac{\# \mathcal{I}_{m}^{\prime}}{T^{m}} \cdot \delta_{0} \\
& +\frac{\# \mathcal{I}_{m}^{\prime \prime}}{T^{m}} \cdot \log \frac{1}{T} \sum_{j=1}^{T} \frac{\left\|B_{j} \cdot B_{\mathbf{i}}\right\|_{1}}{\left\|B_{\mathbf{i}}\right\|_{1}} \\
= & n_{0} \cdot \log \frac{8}{3}-T^{-\left(k_{0}+1\right)} \cdot \delta_{0},
\end{aligned}
$$

where in the inequality we used (114).

3.5. The proof of Theorem 9. It follows from Corollary 17 that to prove our Theorem 9 it is enough to check that

$$
\gamma<\log \frac{8}{3}
$$

where the Lyapunov exponent $\gamma$ was defined in (21). To do so we need to use a theorem of Furstenberg about the integral representation of the Lyapunov exponent. Now we use the definitions from [17, Chapter 3].

Definition 48. We recall that a non-negative $K \times K$ matrix is called column allowable if every column contains a non-zero element. Since we will use this theory for matrices which are the product of $A_{0}, A_{1}, A_{2}$ in the sequel we will always be working with column allowable matrices. We write $\mathcal{C} \mathcal{A}$ for the set of $K \times K$ nonnegative, column allowable matrices. Further let

(117) $\mathcal{C} \mathcal{A}_{p}:=\{A \in \mathcal{C} \mathcal{A}$ : if $\mathbf{r}$ is a row vector of $A$, then either $\mathbf{r}=\mathbf{0}$ or $\mathbf{r}>\mathbf{0}\}$.

For the vectors with all elements positive, $\mathbf{x}=\left(x_{1}, \ldots, x_{K}\right)>\mathbf{0}$ and $\mathbf{y}=$ $\left(y_{1}, \ldots, y_{K}\right)>\mathbf{0}$, we define the pseudo-metric

$$
d(\mathbf{x}, \mathbf{y}):=\log \left[\frac{\max _{i}\left(x_{i} / y_{i}\right)}{\min _{j}\left(x_{j} / y_{j}\right)}\right] .
$$

This is not exactly a metric because $d(\mathbf{x}, \mathbf{y})=0$ if and only if $\mathbf{x}=\lambda \mathbf{y}$ for some real number $\lambda$, but $d$ defines a metric on

$$
\Delta:=\left\{\mathbf{x}=\left(x_{1}, \ldots, x_{K}\right) \in \mathbb{R}^{K}: x_{i}>0 \text { and } \sum_{i=1}^{K} x_{i}=1\right\} .
$$

We call it projective distance. For all $A \in \mathcal{C} \mathcal{A}$ we define

$$
\widetilde{A}: \Delta \rightarrow \Delta \quad \widetilde{A}(\mathbf{x}):=\frac{\mathbf{x}^{T} \cdot A}{\left\|\mathbf{x}^{T} \cdot A\right\|_{1}} .
$$


Finally for any $A \in \mathcal{C} \mathcal{A}$ the Birkhoff contraction coefficient $\tau_{B}(A)$ is defined as the Lipschitz constant for $\widetilde{A}$. That is,

$$
\tau(A):=\sup _{\mathbf{x}, \mathbf{y} \in \Delta, \mathbf{x} \neq \mathbf{y}} \frac{d\left(\mathbf{x}^{T} \cdot A, \mathbf{y}^{T} \cdot A\right)}{d(\mathbf{x}, \mathbf{y})} .
$$

Lemma 49 ([17]). $\quad$ (a): For every $i=1, \ldots, T$ we have $\tau\left(B_{i}\right) \leq 1$.

(b): The map $B_{1}$ is a strict contraction in the projective distance. That is,

$$
h:=\tau\left(B_{1}\right)<1 .
$$

Proof. Part [a]: Since $B_{i} \in \mathcal{C} \mathcal{A}$ the statement can be checked easily. Also it appears as formula [17, (3.1)].

Part [b]: Note that $B_{1} \in \mathcal{C A}_{p}$. Therefore we can use [17, Theorem 3.10] and [17, Theorem 3.12], which yields the assertion.

We will need the following notation from [10]:

Definition 50. On the complete metric space $(\Delta, d)$ we write $M(\Delta)$ for the set of all probability measures on $\Delta$ for which $\mu(\phi)<\infty$ holds for all real-valued Lipschitz functions $\phi$ defined on $(\Delta, d)$. After Hutchinson we define the distance of $\mu, \nu \in M(\Delta)$ by

$$
L(\mu, \nu):=\sup \{\mu(\phi)-\nu(\phi) \mid \phi: \Delta \rightarrow \mathbb{R}, \operatorname{Lip}(\phi) \leq 1\} .
$$

We will consider the metric space $(M(\Delta), L)$. Using that $(\Delta, d)$ is a complete metric space, the main result of [10] implies that

Proposition 51 (Kravchenko [10, Theorem 4.2]). The metric space $(M(\Delta), L)$ is complete.

We introduce the operator $\mathcal{F}: M(\Delta) \rightarrow M(\Delta)$ :

$$
\mathcal{F} \nu(H):=\frac{1}{T} \cdot \sum_{i=1}^{T} \nu\left(\widetilde{B}_{i}^{-1}(H)\right)
$$

for a Borel set $H \subset \Delta$. Using $\nu \in M(\Delta)$, for every Lipschitz function $\phi$ we have

$$
\mathcal{F} \nu(\phi)=\frac{1}{T} \cdot \sum_{i=1}^{T} \nu\left(\phi \circ \widetilde{B}_{i}\right) .
$$

Lemma 52. (a): $\mathcal{F}$ is a contraction on the metric space $(M(\Delta), L)$.

(b): There is a unique fixed point $\nu \in M(\Delta)$ of $\mathcal{F}$ and for all $\mu \in \mathcal{F}$ we have $L\left(\nu, \mathcal{F}^{n} \mu\right) \rightarrow 0$.

Proof of (a). Note that our IFS $\left\{\widetilde{B}_{i}\right\}_{i=1}^{T}$ is not contracting, only contracting on average (that is, the arithmetic mean of the Lipschitz constants of the functions in the IFS is less than one). Let $z:=T /(h+(T-1))>1$. Fix an arbitrary $\phi \in \operatorname{Lip}(1)$. We write

$$
\psi:=\frac{z}{T} \cdot\left(\phi \circ \widetilde{B}_{1}+\cdots+\phi \circ \widetilde{B}_{T}\right) .
$$


Using Lemma 49 we obtain that also $\psi \in \operatorname{Lip}(1)$. Then for an arbitrary $\nu, \mu \in M(\Delta)$ we have

$$
z \cdot[\mathcal{F} \nu(\phi)-\mathcal{F} \mu(\phi)]=\nu(\psi)-\mu(\psi) \leq L(\nu, \mu) .
$$

So, we have obtained that

$$
\forall \mu, \nu \in M(\Delta), \quad L(\mathcal{F} \nu, \mathcal{F} \mu) \leq \frac{1}{z} \cdot L(\nu, \mu),
$$

which completes the proof of Part (a).

Proof of (b). This follows from Proposition 51 and the Banach Fixed Point Theorem. (A contraction on a complete metric space has a unique fixed point.)

From now on we always write $\nu \in M(\Delta)$ for the unique fixed point of the operator $\mathcal{F}$ on $M(\Delta)$. That is,

$$
\nu(\phi)=\frac{1}{T^{n}} \cdot \sum_{i_{1} \ldots i_{n}} \nu\left(\phi \circ \widetilde{B}_{i_{1} \ldots i_{n}}\right)
$$

holds for all Lipschitz functions $\phi$ and $n \geq 1$. Following the idea of Furstenberg [6], it is a key point of our argument that we would like to give an integral representation of the Lyapunov exponent $\gamma_{B}$ as an integral of a function $\varphi$ to be introduced below against the measure $\nu$.

Definition 53. Let $\varphi: \Delta \rightarrow \mathbb{R}$ be defined by

$$
\varphi(\mathbf{x}):=\frac{1}{T} \cdot \sum_{k=1}^{T} \log \left\|\mathbf{x} \cdot B_{k}\right\|_{1}, \quad \mathbf{x} \in \Delta .
$$

Lemma 54. We have $\operatorname{Lip}(\varphi) \leq 1$ on the metric space $(\Delta, d)$.

Proof. Let $\mathbf{x}, \mathbf{y} \in \Delta$. We assume that $\mathbf{x} \neq \mathbf{y}$. Then

$$
\min _{j} \frac{x_{j}}{y_{j}}<1 \text { and } \max _{i} \frac{x_{i}}{y_{i}}>1,
$$

since $\sum x_{i}=\sum y_{i}=1, x_{i}, y_{i}>0$. We fix $k$ with $1 \leq k \leq T$. It is enough to prove that the function

$$
\psi_{k}: \mathbf{x} \mapsto \log \left\|\mathbf{x} \cdot B_{k}\right\|_{1}
$$

is in $\operatorname{Lip}(1)$ because $\varphi$ is the arithmetic mean of the functions $\left\{\psi_{k}\right\}_{k=1}^{T}$. Now fixing $\mathbf{x}, \mathbf{y} \in \Delta, \mathbf{x} \neq \mathbf{y}$

$$
\psi_{k}(\mathbf{x})-\psi_{k}(\mathbf{y})=\log \frac{\left\|\mathbf{x} \cdot B_{k}\right\|_{1}}{\left\|\mathbf{y} \cdot B_{k}\right\|_{1}} .
$$

Note that for arbitrary $\mathbf{a} \in \Delta$ we have

$$
\left\|\mathbf{a} \cdot B_{k}\right\|_{1}=a_{1} \cdot\left\|\mathbf{r}_{1}\right\|_{1}+\cdots+a_{K} \cdot\left\|\mathbf{r}_{K}\right\|_{1},
$$

where $\mathbf{r}_{1}, \ldots, \mathbf{r}_{K}$ are the row vectors of the matrix $B_{k}$. This is so because the matrix $B_{k}$ is non-negative. Hence from (124) we obtain

$$
\psi_{k}(\mathbf{x})-\psi_{k}(\mathbf{y})=\log \frac{\sum_{i=1}^{K} x_{i} \cdot\left\|\mathbf{r}_{i}\right\|_{1}}{\sum_{i=1}^{K} y_{i} \cdot\left\|\mathbf{r}_{i}\right\|_{1}} .
$$


Further, for all $1 \leq i \leq K$, we have

$$
\min _{\ell} \frac{x_{\ell}}{y_{\ell}} \cdot y_{i} \cdot\left\|\mathbf{r}_{i}\right\|_{1} \leq x_{i} \cdot\left\|\mathbf{r}_{i}\right\|_{1} \leq \max _{\ell} \frac{x_{\ell}}{y_{\ell}} \cdot y_{i} \cdot\left\|\mathbf{r}_{i}\right\|_{1} .
$$

Thus, using $y_{i}>0$ for all $1 \leq i \leq K$, we obtain

$$
\min _{\ell} \frac{x_{\ell}}{y_{\ell}} \leq \frac{\sum_{i=1}^{K} x_{i} \cdot\left\|\mathbf{r}_{i}\right\|_{1}}{\sum_{i=1}^{K} y_{i} \cdot\left\|\mathbf{r}_{i}\right\|_{1}} \leq \max _{\ell} \frac{x_{\ell}}{y_{\ell}}
$$

Putting this and (126) together yields that

$$
\log \min _{\ell} \frac{x_{\ell}}{y_{\ell}} \leq \psi_{k}(\mathbf{x})-\psi_{k}(\mathbf{y}) \leq \log \max _{\ell} \frac{x_{\ell}}{y_{\ell}}
$$

From this and (123) we get

$$
\left|\psi_{k}(\mathbf{x})-\psi_{k}(\mathbf{y})\right| \leq \log \max _{\ell} \frac{x_{\ell}}{y_{\ell}}-\log \min _{\ell} \frac{x_{\ell}}{y_{\ell}}=\log \frac{\max _{\ell} \frac{x_{\ell}}{y_{\ell}}}{\min _{\ell} \frac{x_{\ell}}{y_{\ell}}}=d(\mathbf{x}, \mathbf{y}) .
$$

This implies that

$$
|\varphi(\mathbf{x})-\varphi(\mathbf{y})| \leq \frac{1}{T} \sum_{k=1}^{T}\left|\psi_{k}(\mathbf{x})-\psi_{k}(\mathbf{y})\right| \leq d(\mathbf{x}, \mathbf{y})
$$

which completes the proof of the lemma.

Proof of Theorem 9. Using (84) and (116) the only thing left to prove is that

$$
\gamma_{B}<n_{0} \cdot \log \frac{8}{3}
$$

Let

$$
\mathbf{w}:=\frac{1}{K} \cdot \mathbf{e}, \text { where } \mathbf{e}:=(1, \ldots, 1) \in \mathbb{R}^{K} .
$$

We define the sequence of measures $\nu_{n} \in \mathcal{M}^{1}$ by

$$
\nu_{0}:=\delta_{\mathbf{w}} \text { and } \nu_{n}(H):=\left(\mathcal{F}^{n} \nu_{0}\right)(H)=\frac{1}{T^{n}} \cdot \sum_{i_{1} \ldots i_{n}} \nu_{0}\left(\widetilde{B}_{i_{1} \ldots i_{n}}^{-1}(H)\right),
$$


where $H \subset \Delta$ is a Borel set. Observe that

$$
\begin{aligned}
\int_{\Delta} \varphi(\mathbf{x}) d \nu_{n}(\mathbf{x}) & =\frac{1}{T^{n}} \sum_{i_{1} \ldots i_{n}} \int_{\Delta} \varphi(\mathbf{x}) d \nu_{0}\left(\widetilde{B}_{i_{n} \ldots i_{1}}^{-1}(\mathbf{x})\right) \\
& =\frac{1}{T^{n}} \sum_{i_{1} \ldots i_{n}} \varphi\left(\widetilde{B}_{i_{n} \ldots i_{1}}(\mathbf{w})\right) \\
& =\frac{1}{T} \sum_{k=1}^{T} \frac{1}{T^{n}} \sum_{i_{1} \ldots i_{n}} \log \left\|\widetilde{B}_{i_{n} \ldots i_{1}}(\mathbf{w}) \cdot B_{k}\right\|_{1} \\
& =\frac{1}{T} \sum_{k=1}^{T} \frac{1}{T^{n}} \sum_{i_{1} \ldots i_{n}} \log \frac{\left\|\mathbf{w} \cdot B_{i_{1} \ldots i_{n}} \cdot B_{k}\right\|_{1}}{\left\|\mathbf{w} \cdot B_{i_{1} \ldots i_{n}}\right\|_{1}} \\
& =\frac{1}{T^{n+1}} \sum_{i_{1} \ldots i_{n+1}} \log \left\|\mathbf{w} \cdot B_{i_{1} \ldots i_{n}+1}\right\|_{1}-\frac{1}{T^{n}} \sum_{i_{1} \ldots i_{n}} \log \left\|\mathbf{w} \cdot B_{i_{1} \ldots i_{n}}\right\|_{1} \\
& =\frac{1}{T^{n}} \cdot \sum_{i_{1} \ldots i_{n}} \frac{1}{T} \log \prod_{k=1}^{T} \frac{\left\|\mathbf{w} \cdot B_{k} \cdot B_{i_{1} \ldots i_{n}}\right\|_{1}}{\left\|\mathbf{w} \cdot B_{i_{1} \ldots i_{n}}\right\|_{1}} \\
& =\frac{1}{T^{n}} \cdot \sum_{i_{1} \ldots i_{n}} \frac{1}{T} \log \prod_{k=1}^{T} \frac{\left\|B_{k} \cdot B_{i_{1} \ldots i_{n}}\right\|_{1}}{\left\|B_{i_{1} \ldots i_{n}}\right\|_{1}} .
\end{aligned}
$$

Using Lemma 47 we obtain that for all $n \geq k_{0}+1$,

$$
\int_{\Delta} \varphi(\mathbf{x}) d \nu_{n}(\mathbf{x}) \leq n_{0} \cdot \log \frac{8}{3}-T^{-\left(k_{0}+1\right)} \cdot \delta_{0} .
$$

In the rest of the proof we will verify that the limit of the left hand side is equal to $\gamma_{B}$, from which it immediately follows that (129) holds. Namely, results of Furstenberg [6] indicate that the Lyapunov exponent $\gamma_{B}$ (defined in (83)) has the following integral representation:

$$
\gamma_{B}=\int_{\Delta} \varphi(\mathbf{x}) d \nu(\mathbf{x})=\lim _{n \rightarrow \infty} \int_{\Delta} \varphi(\mathbf{x}) d \nu_{n}(\mathbf{x}),
$$

where the second equality follows from Lemma 52, Although we believe that this follows from [6], for the convenience of the reader we give a short proof of (132) here.

We start with an easy observation: in (130) we put together the left hand side of the first line and the right side of the fifth line. Then we apply for both $\ell=n+1$ and $\ell=n$ that

$$
\frac{1}{T^{\ell}} \sum_{i_{1} \ldots i_{\ell}} \log \left\|\mathbf{w} \cdot B_{i_{1} \ldots i_{\ell}}\right\|_{1}=\log 1 / K+\frac{1}{T^{\ell}} \sum_{i_{1} \ldots i_{\ell}} \log \left\|B_{i_{1} \ldots i_{\ell}}\right\|_{1}
$$

to obtain

$$
\int_{\Delta} \varphi(\mathbf{x}) d \nu_{n}(\mathbf{x})=\frac{1}{T^{n+1}} \sum_{i_{1} \ldots i_{n+1}} \log \left\|B_{i_{1} \ldots i_{n+1}}\right\|_{1}-\frac{1}{T^{n}} \sum_{i_{1} \ldots i_{n}} \log \left\|B_{i_{1} \ldots i_{n}}\right\|_{1} .
$$

Let us define the measure

$$
\eta_{\ell}:=\frac{1}{\ell} \sum_{n=1}^{\ell} \nu_{n} .
$$


Clearly $\eta_{\ell} \in M(\Delta)$ holds for all $\ell$ and it follows from Lemma 52 that for all Lipschitz functions $\phi$ we have

$$
\lim _{\ell \rightarrow \infty} \eta_{\ell}(\phi)=\lim _{n \rightarrow \infty} \nu_{n}(\phi)=\nu(\phi) .
$$

Note that by (133) the integral $\eta_{\ell}(\varphi)$ is given by a telescopic sum. This yields

$$
\eta_{\ell}(\varphi)=\frac{1}{\ell} \sum_{n=1}^{\ell} \nu_{n}(\varphi)=\frac{1}{\ell}\left(\frac{1}{T^{\ell+1}} \sum_{i_{1} \ldots i_{\ell+1}} \log \left\|B_{i_{1} \ldots i_{\ell+1}}\right\|_{1}-\frac{1}{T} \cdot \sum_{i=1}^{T} \log \left\|B_{i}\right\|_{1}\right) .
$$

Now the assertion of (132) immediately follows from (85). As was noted above, (129) follows from (131) and (132), which completes the proof of Theorem 9

\section{The Remaining PRoofs}

The angle $\theta$ projection from $S$ to the $y$-axis is denoted by $\mathrm{PROJ}^{\theta}$. We are using notation defined in Section 3.1. The $\operatorname{proj}^{\theta}$ projection of the IFS $\mathcal{G}:=$ $\left\{g_{i}(x, y)=\frac{1}{3}(x, y)+\frac{1}{3} \mathbf{t}_{i}\right\}_{i=1}^{8}$ is the IFS

$$
\Phi:=\left\{\varphi_{i}^{\theta}(t)=\frac{1}{3} \cdot t+\frac{1}{3} \cdot \operatorname{proj}^{\theta}\left(\mathbf{t}_{i}\right)\right\}_{i=1}^{8} .
$$

That is, for every $i=1, \ldots, 8$ the following diagram is commutative:

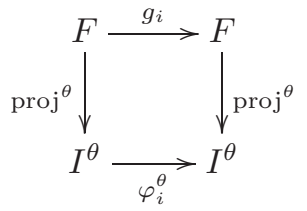

4.1. The measures $\nu^{\theta}$ and $\widetilde{\nu}^{\theta}$. We recall that, for $\theta \in[0, \pi / 2)$ and $a \in I^{\theta}$, Line $_{\theta, a}$ denotes the line segment in $[0,1]^{2}$ of angle $\theta$ through the point $(0, a)$. Further, for any $a \in[0,1]$, let $\operatorname{LINE}_{\theta, a}$ be the line segment in $S$ of angle $\theta$ through the point $(0, a)$. Using the notation of Definition 19 we define the measure $\widetilde{\nu}$ on the Borel subsets of $\widetilde{F}$ as follows:

$$
\widetilde{\nu}:=\left.\frac{1}{N} \sum_{i=1}^{K}\left(\left(q_{i}, r_{i}\right)+\nu\right)\right|_{S} .
$$

The angle $\theta$ projection of $\widetilde{\nu}$ to the $y$-axis is called $\widetilde{\nu}^{\theta}$. When $\pi^{\theta}:=\operatorname{proj}^{\theta} \circ \Pi$, this can be summarized by the following diagram is commutative:

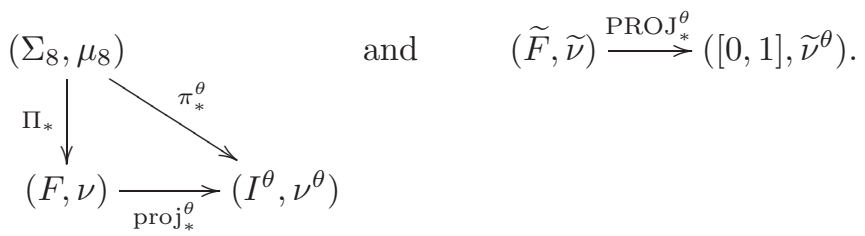

It is immediate from the definitions and from the symmetries of the Sierpinski carpet that for Borel sets $H \subset[-\tan \theta, 0]$ and $P \subset[0,1]$ we have

$$
\widetilde{\nu}^{\theta}(P)=\nu^{\theta}(H)=0 \text { implies that } \nu^{\theta}(P)=0 \text { and } \nu^{\theta}(1-\tan \theta-H)=0 .
$$


On the other hand, for $H \subset[-\tan \theta, 0], \nu^{\theta}(H)=0$ does not imply that $\nu^{\theta}(1+H)=$ 0 . This makes our investigation more complicated than the analogous proof for the Lebesgue measure. We will use the following observation.

Lemma 55. The measure $\widetilde{\nu}^{\theta}$ is invariant under the map $y \mapsto 3 y \bmod 1$.

Proof. For $\left(i_{1}, \ldots, i_{n}\right) \in\{0,1,2\}^{n}$ we write

$$
I_{i_{1} \ldots i_{n}}:=\left[\sum_{k=1}^{n} i_{k} \cdot 3^{-k}, \sum_{k=1}^{n} i_{k} \cdot 3^{-k}+3^{-n}\right] .
$$

Since the $\nu$ measure of every level $n$ shape $j$ is $8^{-n} \nu\left(Q_{j}\right)$ we obtain

$$
\widetilde{\nu}^{\theta}\left(I_{i_{1} \ldots i_{n}}\right)=\sum_{j=1}^{K} \#_{i_{1} \ldots i_{n}}(j) \cdot 8^{-n} \nu\left(Q_{j}\right),
$$

where $\#_{i_{1} \ldots i_{n}}(j)$ denotes the number of level $n$ shapes $j$ contained in $\widetilde{F}^{n} \cap S_{i_{1} \ldots i_{n}}$. That is,

$$
\#_{i_{1} \ldots i_{n}}(j)=\sum_{k=1}^{K} A_{i_{1} \ldots i_{n}}(k, j) .
$$

Since from (19) all the column sums of the matrix $A_{0}+A_{1}+A_{2}$ are equal to 8, this readily implies that

$$
\sum_{i_{0}=0}^{2} \#_{i_{0} i_{1} \ldots i_{n}}(j)=8 \cdot \#_{i_{1} \ldots i_{n}}(j) .
$$

Since the inverse image by $y \mapsto 3 y \bmod 1$ of $I_{i_{1} \ldots i_{n}}$ is $\bigcup_{i_{0}=0}^{2} I_{i_{0} i_{1} \ldots i_{n}}$, formulae (136) and (138) together imply the assertion of the lemma.

\subsection{The dimension of the intersection is a constant $\nu^{\theta}$-almost surely.}

Lemma 56. For every $\theta \in[0, \pi)$, the following functions are $\mu_{8}$-almost everywhere constant:

$$
\mathbf{i} \mapsto \operatorname{dim}_{\mathrm{H}}\left(E_{\theta, \pi^{\theta}(\mathbf{i})}\right), \mathbf{i} \mapsto \overline{\operatorname{dim}}_{\mathrm{B}}\left(E_{\theta, \pi^{\theta}(\mathbf{i})}\right), \mathbf{i} \mapsto \underline{\operatorname{dim}}_{\mathrm{B}}\left(E_{\theta, \pi^{\theta}(\mathbf{i})}\right) .
$$

Proof. The fact that these functions are Borel measurable can be proved in exactly the same way as the analogous statement proved in [9, Lemma 2.4]. Now, for every $\mathbf{i} \in \Sigma_{8}$ and $k \in\{1, \ldots, 8\}$, we have $g_{k}\left(\right.$ Line $\left._{\theta, \pi^{\theta}(\mathbf{i})}\right) \subset$ Line $_{\theta, \pi^{\theta}(k \mathbf{i})}$, which implies that these three functions have the property

$$
\forall \mathbf{j} \in \Sigma, \quad f(\sigma \mathbf{j}) \leq f(\mathbf{j}) .
$$

Such a function is $\mu_{8}$-almost everywhere constant since $\left(\sigma, \mu_{8}\right)$ is ergodic and, for each $t, \sigma\{\mathbf{i}: f(\mathbf{i}) \leq t\} \subset\{\mathbf{i}: f(\mathbf{i}) \leq t\}$.

Clearly, for every $\theta, a$,

$$
\text { if } \Pi(\mathbf{i}), \Pi(\mathbf{j}) \in E_{\theta, a} \text {, then } \operatorname{dim}\left(E_{\theta, \pi^{\theta}(\mathbf{i})}\right)=\operatorname{dim}\left(E_{\theta, \pi^{\theta}(\mathbf{j})}\right)
$$

if dim means any one of Hausdorff dimension, lower box dimension or upper box dimension.

Proof of Proposition 3 . The assertion of Proposition 3 follows immediately from (141) and Lemma 56 
The proof of Theorem 7 is based on a method of Kenyon-Peres which uses a theorem due to Ledrappier (see [9, Proposition 2.6]). We use their method but we have to tackle an additional problem: unlike the Lebesgue measure, our measure $\nu^{\theta}$ is not translation invariant, which makes this case much more difficult.

Proof of Proposition 8. In this proof when we write dim it means any of the following dimensions: $\operatorname{dim}_{\mathrm{H}}, \underline{\operatorname{dim}}_{\mathrm{B}}$ or $\overline{\operatorname{dim}}_{\mathrm{B}}$. By Lemma 55 we can apply [9, Proposition 2.6] exactly as in the proof of [11, Theorem 1.1 (3)] to obtain

$$
\text { for } \widetilde{\nu}^{\theta} \text {-almost all } b \in[0,1], \operatorname{dim}_{\mathrm{H}}\left(\operatorname{LINE}_{\theta, b} \cap \widetilde{F}\right)=\operatorname{dim}_{\mathrm{B}}\left(\operatorname{LINE}_{\theta, b} \cap \widetilde{F}\right)
$$

and the box dimension exists.

We fix $\mathbf{a}:=\left(a_{1}, \ldots, a_{n_{0}}\right) \in\{0,1,2\}^{n_{0}}$ as constructed in Proposition 18, We use the definitions of Section 3.2.1 (see Figure 4). For every $j \in\{1, \ldots, K\}$, in $S_{\mathbf{a}}$ there are altogether $3^{n_{0}}$ level $n$ shapes $j$. These were denoted (see (36)) by

$$
Q_{\mathbf{a}}^{\bar{\ell}}(j): \bar{\ell}=\left(\ell_{1}, \ldots, \ell_{n_{0}}\right) \in\{0,1,2\}^{n_{0}} .
$$

When we consider $\widetilde{F}^{n_{0}} \cap S_{\mathbf{a}}$ some of these shapes are completely deleted by the construction of the $n_{0}$ approximation $\widetilde{F}^{n_{0}}$ of the translated copies of the Sierpinski carpet while some other level $n_{0}$ shapes are preserved completely by this construction. Thus for every $j \in\{1, \ldots, K\}$ we can define the set of indices $\mathcal{I}_{1}(j) \subset \mathcal{I}(j) \subset\{0,1,2\}^{n_{0}}$ such that

$$
\widetilde{F}^{n_{0}} \cap S_{\mathbf{a}}=\bigcup_{j=1}^{K} \bigcup_{\bar{\ell} \in \mathcal{I}(j)} Q_{\mathbf{a}}^{\bar{\ell}}(j) \text { and } \widetilde{F}^{n_{0}} \cap S_{\mathbf{a}} \cap Q_{1}=\bigcup_{j=1}^{K} \bigcup_{\bar{\ell} \in \mathcal{I}_{1}(j)} Q_{\mathbf{a}}^{\bar{\ell}}(j) \text {. }
$$

It follows from the special choice of a that we have

$$
\mathcal{I}_{1}(j) \neq \emptyset \text {. }
$$

By self-similarity for every $b \in[0,1]$ and $1 \leq j \leq K$,

$$
\text { if } Q_{\mathbf{a}}^{\bar{\ell}}(j) \subset \widetilde{F}^{n} \text {, then } \operatorname{dim}\left(\operatorname{LINE}_{\theta, b} \cap \widetilde{F} \cap Q_{\mathbf{a}}^{\bar{\ell}}(j)\right)=\operatorname{dim}\left(\operatorname{LINE}_{\theta, 3^{n}{ }_{0} b} \cap Q_{j} \cap \widetilde{F}\right) \text {, }
$$

where $3^{n_{0}} b$ is written for $3^{n_{0}} b \bmod 1$. Using (144) and (145) we see that

for every $b \in I_{\mathbf{a}}:=\left[\sum_{k=1}^{n_{0}} a_{k} 3^{-k}, \sum_{k=1}^{n_{0}} a_{k} 3^{-k}+3^{-n_{0}}\right]$ we have

$$
\operatorname{dim}\left(E_{\theta, b}\right)=\max _{j} \operatorname{dim}\left(\operatorname{LINE}_{\theta, 3^{n_{0}} b} \cap Q_{j}\right)=\operatorname{dim}\left(\operatorname{LINE}_{\theta, b} \cap \widetilde{F}\right) .
$$

Using this, (142) and (135) we obtain that for $\nu^{\theta}$-almost all $b \in I_{\mathbf{a}}$ we have

$$
\operatorname{dim}_{\mathrm{H}}\left(E_{\theta, b}\right)=\operatorname{dim}_{\mathrm{B}}\left(E_{\theta, b}\right)
$$

and the box dimension exists. Clearly, $\nu^{\theta}\left(I_{\mathbf{a}}\right)>0$. This and Proposition 3 together imply that the conclusion of Proposition 8 holds.

4.3. The local dimension of $\nu^{\theta}$ and the box dimension of the slices. Let $\theta \in[0, \pi / 2)$ be arbitrary. For $a \in I^{\theta}$, let $N_{\theta, a}(n)$ be the number of level $n$ squares of $F^{n}$ that intersect $E_{\theta, a}$. By definition,

$$
\underline{\operatorname{dim}}_{\mathrm{B}}\left(E_{\theta, a}\right)=\liminf _{r \rightarrow 0} \frac{\log N_{\theta, a}(n)}{n \log 3} \text { and } \overline{\operatorname{dim}}_{\mathrm{B}}\left(E_{\theta, a}\right)=\limsup _{r \rightarrow 0} \frac{\log N_{\theta, a}(n)}{n \log 3} .
$$


We write $\operatorname{Line}_{\theta, a}\left(3^{-n}\right)$ for the $3^{-n}$ vertical neighborhood of Line $\theta_{\theta, a}$. That is,

$$
\text { Line }_{\theta, a}\left(3^{-n}\right):=\left\{(x, y):|y-(x \cdot \tan \theta+a)|<3^{-n}, x \in[0,1]\right\} .
$$

For arbitrary $n$ we shall estimate the $\nu$ measure of $\operatorname{Line}_{\theta, a}\left(3^{-n}\right)$ by the $\nu$ measure of those squares of level $n$ or close to $n$ that intersect $\operatorname{Line}_{\theta, a}$. First we fix $k_{0}$ such that $1+\tan \theta<3^{k_{0}}$ and observe that if a level $n+k_{0}$ square from $F^{n+k_{0}}$ intersects $E_{\theta, a}$, then this square is contained in $\operatorname{Line}_{\theta, a}\left(3^{-n}\right)$. Since the $\nu$ measure of such a square is equal to $8^{-\left(n+k_{0}\right)}$ we see that

$$
\forall \theta, a: \quad 8^{-\left(n+k_{0}\right)} \cdot N_{\theta, a}\left(n+k_{0}\right) \leq \nu\left(\operatorname{Line}_{\theta, a}\left(3^{-n}\right)\right) .
$$

Now we prove an opposite inequality.

Lemma 57. Let $\theta \in[0, \pi / 2)$ and $a \in I^{\theta}$ be arbitrary. We have

$$
\nu\left(\text { Line }_{\theta, a}\left(3^{-n}\right)\right)<1000 \cdot\left(\left\lfloor\frac{1}{\tan \theta}\right\rfloor+1\right) \cdot 8^{-n} \cdot N_{\theta, a}(n) .
$$

Proof. In this proof we write $G_{\theta, a}(n)$ for the collection of those "good" level $n$ squares from $F^{n}$ that intersect $E_{\theta, a}$. Further we write $B_{\theta, a}(n)$ for the collection of those "bad" level $n$ squares from $F^{n}$ that do not intersect $E_{\theta, a}$ but do intersect Line $_{\theta, a}\left(3^{-n}\right)$. Since all the level $n$ squares from $F^{n}$ have $\nu$-measure $8^{-n}$, to prove this lemma it is enough to show that

$$
\# B_{\theta, a}(n)<1000 \cdot\left(\left\lfloor\frac{1}{\tan \theta}\right\rfloor+1\right) \cdot \# G_{\theta, a}(n) .
$$

Choose $U \in B_{\theta, a}(n)$. Without loss of generality we may assume that $U$ is situated below the line Line $_{\theta, a}$. Write

$$
U^{W}, U^{W^{2}}, \ldots, U^{W^{k}}
$$

for the sequence of consecutive level $n$ squares on the left of $U$ with $k$ chosen so that $U^{W^{k}}$ is the first that intersects $\operatorname{Line}_{\theta, a}$. Then $k \leq\left\lfloor\frac{1}{\tan \theta}\right\rfloor+1$. If we write $U^{N}$ for the level $n$ square above $U$ and

$$
U^{N}, U^{N W}, \ldots, U^{N W^{k}}
$$

for the consecutive level $n$ squares to the left of $U^{N}$, all of which meet line Line ${ }_{\theta, a}$, then either one of them, $V$ say, is in $F^{n}$ and we will say that $V$ is associated with $U$ or none of them is in $F^{n}$. This is possible only if there is $\ell<n$ such that $3^{-\ell}>k \cdot 3^{-n}$ and a level $\ell$ square, let us call it $X$, which is situated in such a way that its bottom line contains the bottom lines of all the squares from (152). Then the level $\ell$ square $X^{S}$ below $X$ is in $F^{\ell}$ and the level $n$ squares in (1510 are in the top row in $X^{S}$ and lie in $F^{n}$. Then $U^{W^{k}}$ belongs to $G_{\theta, a}(n)$ and we say that it is associated to $U$. In this way we have seen that for every "bad" square $U$ there is an associated "good" square $V$ within distance $\left(\left\lfloor\frac{1}{\tan \theta}\right\rfloor+1\right) \cdot 3^{-n}$. It follows that all the elements of $G_{\theta, a}(n)$ can be associated with less than $1000 \cdot\left(\left\lfloor\frac{1}{\tan \theta}\right\rfloor+1\right)$ squares from $B_{\theta, a}(n)$, which completes the proof of the lemma.

Proof of Proposition 4. Putting formulas (148) and (149) together we obtain that for all $\theta \in[0, \pi / 2)$ we have

$$
8^{k_{0}} N_{\theta, a}\left(n+k_{0}\right) \leq 8^{n} \nu^{\theta}\left(\left[a-3^{-n}, a+3^{-n}\right]\right) \leq k_{1} N_{\theta, a}(n),
$$


where

$$
k_{0}=\left\lfloor\frac{\log (1+\tan \theta)}{\log 3}\right\rfloor+1, \quad k_{1}=1000\left(\left\lfloor\frac{1}{\tan \theta}\right\rfloor+1\right) .
$$

Taking logarithms and dividing by $\log 3^{n}$ we obtain

$$
s-o(n) \leq \frac{\log N_{\theta, a}(n)}{\log 3^{n}}+\frac{\log \nu^{\theta}\left(\left[a-3^{-n}, a+3^{-n}\right]\right)}{\log 3^{-n}} \leq s+o(n) .
$$

Proof of Theorem 5. The assertion immediately follows from (3) and Proposition 4 since $\operatorname{dim}_{\mathrm{H}}\left(\nu^{\theta}\right) \leq 1$.

Proof of Theorem 11. This is immediate from Theorem 5 and Proposition 8 .

Proof of Corollary 10. This follows from Theorem 9 and Proposition 4 ,

Proof of Theorem 6. We only need to prove part (a). $\nu^{\theta} \sim \mathcal{L}$ eb follows from 16 , Proposition 3.1], which states that every self-similar measure is either equivalent to the Lebesgue measure or singular. Using the argument from [12, p.140] we see that for $\mathcal{L}$ eb almost all $a \in I^{\theta}$ the conditional measure

$$
\nu_{\theta, a}(B):=\lim _{\delta \rightarrow 0} \frac{\nu(B(\delta))}{2 \delta}, \quad B \subset E_{\theta, a} \text { Borel }
$$

exists and is finite and positive from [12, (10.2)]. Now (9) follows from (153).

\section{REFERENCES}

[1] K. Athreya, P. Ney, Branching Processes, Springer, 1972. MR0373040 (51:9242)

[2] P. Bougerol, J. Lacroix, Products of random matrices with applications to Schrödinger operators, Birkhäuser, Basel, 1985. MR886674 (88f:60013)

[3] K. Falconer, Fractal Geometry, 2nd ed., Wiley, 2003. MR2118797(2006b:28001)

[4] D.J. Feng, H.Hu, Dimension theory of iterated function systems, Communications on Pure and Applied Mathematics 62 (2009), 1435-1500. MR2560042 (2010i:37049)

[5] D.J. Feng, N. Sidorov, Growth rate for beta-expansions, Monatsh. Math. 162 (2011), 41-60. MR 2747343

[6] H. Furstenberg, Non-commuting random products, Trans. Amer. Math. Soc. 108 (1963), 377-428. MR0163345 (29:648)

[7] H. Furstenberg, Disjointness in ergodic theory, minimal sets, and a problem in Diophantine approximation, Math. Systems Theory 1 (1967), 1-49. MR0213508 (35:4369)

[8] J. E. Hutchinson, Fractals and self-similarity, Indiana Univ. Math. J. 30 (1981), 713-747. MR625600 (82h:49026)

[9] R. Kenyon, Y. Peres, Intersecting random translates of invariant Cantor sets. Invent. Math. 104 (1991), 601-629. MR1106751 (92g:28018)

[10] A. S. Kravchenko, Completeness of the space of separable measures in the KantorovichRubinshtein metric. Siberian Math. J. 47 (2006), no. 1, 68-76. MR2215298 (2007b:28003)

[11] Qing-Hui Liu, Li-Feng Xi and Yan-Fen Zhao, Dimension of the intersection of the Sierpinski carpet with lines of rational slopes, Proc. Edinb. Math. Soc. 50 (2007), 411-428. MR2334955 (2008i:28014)

[12] P. Mattila, Geometry of sets and measures in Euclidean spaces, Cambridge, 1995. MR $1333890(96 \mathrm{~h}: 28006)$

[13] J.M. Marstrand, Some fundamental geometrical properties of plane sets of fractional dimensions, Proc. Lond. Math. Soc. 4 (1954), 257-302. MR0063439 (16:121g)

[14] H-O. Peitgen, H. Jürgens and D. Saupe, Chaos and Fractals, Springer, New York, 2004. MR2031217 (2004m:37061)

[15] Y. Peres, Domain of analytic continuation for the top Lyapunov exponent, Ann. Inst. H. Poincaré Probab. Statist. 28 (1992), 131-148. MR 1158741 (93g:60012) 
[16] Y. Peres, W. Schlag and B. Solomyak, Sixty years of Bernoulli convolutions, In Fractal Geometry and Stochastics, II (Greifswald/Koserow, 1998), Progr. Probab., Vol. 46, Birkhäuser, Basel, 39-65, 2000. MR 1785620 (2001m:42020)

[17] E. Seneta, Non-negative matrices and Markov chains, Springer, 1981. MR2209438

[18] P. Walters, An introduction to Ergodic Theory, Springer, 1982. MR648108 (84e:28017)

[19] L.S. Young, Dimension entropy and Lyapunov exponents, Ergodic Theory and Dynamical Systems 2 (1982), 109-124. MR684248 (84h:58087)

Mathematics Institute, University of Warwick, Coventry CV4 7AL, United Kingdom E-mail address: A.Manning@warwick.ac.uk

Institute of Mathematics, Technical University of Budapest, H-1529 P.O. Box 91, BudAPEST, Hungary

E-mail address: simon@math.bme.hu 